Check for updates

Cite this: J. Mater. Chem. B, 2020, 8, 8171

Received 17th April 2020,

Accepted 28th July 2020

DOI: 10.1039/d0tb01005g

rsc.li/materials-b

\title{
Multicomponent polysaccharide alginate-based bioinks
}

\author{
Carmen C. Piras (D)* and David K. Smith (DD
}

\begin{abstract}
3D-Bioprinting has seen a rapid expansion in the last few years, with an increasing number of reported bioinks. Alginate is a natural biopolymer that forms hydrogels by ionic cross-linking with calcium ions. Due to its biocompatibility and ease of gelation, it is an ideal ingredient for bioinks. This review focuses on recent advances on bioink formulations based on the combination of alginate with other polysaccharides. In particular, the molecular weight of the alginate and its loading level have an impact on the material's performance, as well as the loading of the divalent metal salt and its solubility, which affects the cross-linking of the gel. Alginate is often combined with other polysaccharides that can sigificantly modify the properties of the gel, and can optimise alginate for use in different biological applications. It is also possible to combine alginate with sacrificial polymers, which can temporarily reinforce the 3D printed construct, but then be removed at a later stage. Other additives can be formulated into the gels to enhance performance, including nanomaterials that tune rheological properties, peptides to encourage cell adhesion, or growth factors to direct stem cell differentiation. The ease of formulating multiple components into alginate gels gives them considerable potential for further development. In summary, this review will facilitate the identification of different alginate-polysaccharide bioink formulations and their optimal applications, and help inform the design of second generation bioinks, allowing this relatively simple gel system to achieve more sophisticated control over biological processes.
\end{abstract}

\section{Introduction}

3D-Bioprinting is a rapidly expanding technology with much promise in tissue engineering and regenerative medicine. ${ }^{1-6}$ This manufacturing technique is based on the 3D deposition of biomaterials incorporating cells in desired layer-by-layer patterns. A variety of applications has been reported for this emerging technology including, amongst others, the production of bone, cartilage and retina scaffolds, endothelial tissue and vascular constructs, cardiac tissue and heart valves, and skin regeneration..$^{7-10}$

One of the most important factors for the successful fabrication of 3D printed scaffolds is the choice of the material to be printed, or bioink. The bioink has to be suitable for the printing technology adopted and has to display the right mechanical properties (e.g. viscosity, stiffness, shear thinning behaviour) to allow both the 3D deposition of the ink and stability of the obtained constructs, but, more importantly, must be biocompatible to ensure cell survival during and after printing. ${ }^{11-13}$

Due to their chemical and physical properties, high water content, biodegradability and capability to mimic the natural

Department of Chemistry, University of York, Heslington, YO10 5DD, UK.

E-mail: carmen.piras@york.ac.uk environment of cells, hydrogels are ideal candidates as bioinks for 3D bioprinting. ${ }^{12}$ These materials can incorporate more than $99 \%$ water and can be obtained from cross-linked polymer gelators (PGs) or via the self-assembly of low molecular weight gelators (LMWGs) in aqueous media. ${ }^{14}$ So far, due to their ease of gelation, simple manipulation and highly tuneable mechanical properties, a wide variety of polymeric hydrogels have been studied as bioinks. The majority of them are based on natural molecules, such as collagen ( $c a .26 \%$ of the reported bioinks) and alginate ( $c a .24 \%$ ) or their composites with other polymers. ${ }^{15}$ Further bioinks reported in literature are based on other natural and synthetic polymers including hyaluronic acid, gelatin, cellulose, soy protein, fibrinogen, chitosan, dextran, starch, polylactic acid (PLA), poly(lactic-co-glycolic) acid (PLGA), polyethylene glycol (PEG), polycaprolactone (PCL). ${ }^{15}$

Alginate is a natural polymer that can easily form hydrogels by simple mixing with divalent cations. Being biocompatible and very versatile, it is an ideal candidate for 3D bioprinting. Since this field has seen a rapid expansion in the last few years, the number of research articles on novel bioink formulations is dramatically increasing. For this reason, when approaching this research field, it can be difficult to acquire a clear idea of the available formulations and their applications. This review focuses on alginate-based bioinks prepared using pure alginate 
or in combination with other polysaccharides. The main goal is to update the reader on the latest developments in this field, providing a simple guide on available formulations, their compositions and biological applications. We hope that this article will simplify the identification of the main types of alginate-based polysaccharidic bioinks and inform the design of novel formulations. By developing a series of 'design principles' for bioprinted alginate gels, we hope that this review will facilitate the development of new formulations with additional functionality and further promote uptake of these fascinating gel-phase materials in high-tech applications.

\section{Alginate-based bioinks and factors that influence the properties of the 3D printed structures}

Alginate is a polysaccharide extracted from brown algae. This biopolymer is composed of $\beta$-D-mannuronic acid and $\alpha$-L-glucuronic acid units linked through $\beta(1-4)$ bonds (Fig. 1). ${ }^{16}$ The sodium salt of alginate is water-soluble and can readily form hydrogels when mixed with multivalent cations $\left(\right.$ e.g. $\mathrm{Ca}^{2+}$, $\mathrm{Ba}^{2+}, \mathrm{Sr}^{2+}$ ) by generating ionic inter-chain bridges. ${ }^{17}$ The ease of gelation at room temperature, the mechanical properties, and biocompatibility of sodium alginate make hydrogels of this polymer great candidates as bioinks. A wide variety of alginate-based inks has been reported and some of them are commercially available (e.g. 'CELLINK'). , $^{8,18,19}$

\section{Overview of alginate $3 \mathrm{D}$ printing}

One of the most advantageous features of alginate is its versatility and applicability to a variety of scaffold fabrication methods (e.g. spheroids, vascular constructs, microfluidic fibreshaped scaffolds $)^{20-23}$ and bioprinting technologies (e.g. extrusion, inkjet, and microfluidic bioprinting). ${ }^{24-27}$ Extrusion is probably the most widely employed bioprinting methodology, employing the controlled extrusion of long hydrogel filaments from a dispensing cartridge. ${ }^{25}$ This technique can be applied with a variety of bioinks ranging from low to high viscosities. Advantages of this methodology include scalability and cost-efficiency, but it has lower resolution compared to other methods. This review will mainly focus on the different alginate/polysaccharide bioink formulations from a biomaterial point of view rather than focus on printing technology. Most of the examples we will discuss (unless specified) are based on extrusion 3D printing, which is the most commonly used technique for alginate/polysaccharide blends. We will explore in detail the factors that can influence the printing process and the

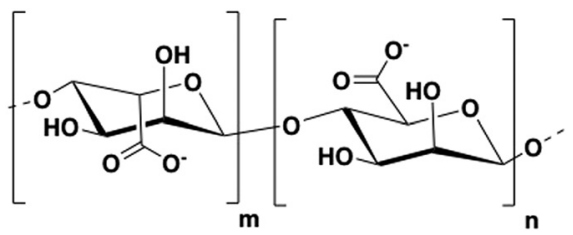

Fig. 1 Chemical structure of alginate. properties of the 3D printed constructs obtained by extrusion. However, because alginate is easy to gel at room temperature, allowing the encapsulation of living cells before the printing process, it can be readily applied to other bioprinting methods. The reader is, therefore also referred to a number of additional reviews and research articles for a more detailed discussion, ${ }^{24,26-34}$ and we will go on to briefly review inkjet and microfluidic bioprinting technologies, for which the properties of this polymer are particularly advantageous, later in this article.

\section{Factors that influence the properties of the 3D printed structures}

Being a natural polymer, different types of sodium alginate are commercially available, with different numbers of repeat units, molecular weights and viscosities. All these factors, together with the polymer concentration in aqueous solution, can influence the features of the resulting hydrogels (e.g. porosity, mechanical properties, shear-thinning behaviour, degradability) and therefore the quality of the corresponding bioinks and their compatibility with the growth of specific cell types (Fig. 2). ${ }^{8,35-39}$ All these elements should therefore be taken into consideration when designing new bioinks.

More than 200 different alginates are currently being manufactured with molecular weights that can vary between 32 and $400 \mathrm{kDa}$, and a different content of $\beta$-D-mannuronic acid (M) and $\alpha$-L-glucuronic acid (G) units. ${ }^{40}$ Chen and co-workers demonstrated that the molecular weight and the ratio between the $\beta$-D-mannuronic acid (M) and $\alpha$-L-glucuronic acid (G) constituent units of sodium alginate can significantly influence the rheological properties of the corresponding aqueous solutions. ${ }^{41}$ Alginate chains of different molecular weight and different $\mathrm{M} / \mathrm{G}$ ratio and were obtained by acid hydrolysis and separated by gel permeation chromatography. The viscosity studies performed on aqueous solutions of the different samples showed that the samples with lower molecular weight and higher number of $\mathrm{M}$ units displayed a higher viscosity than

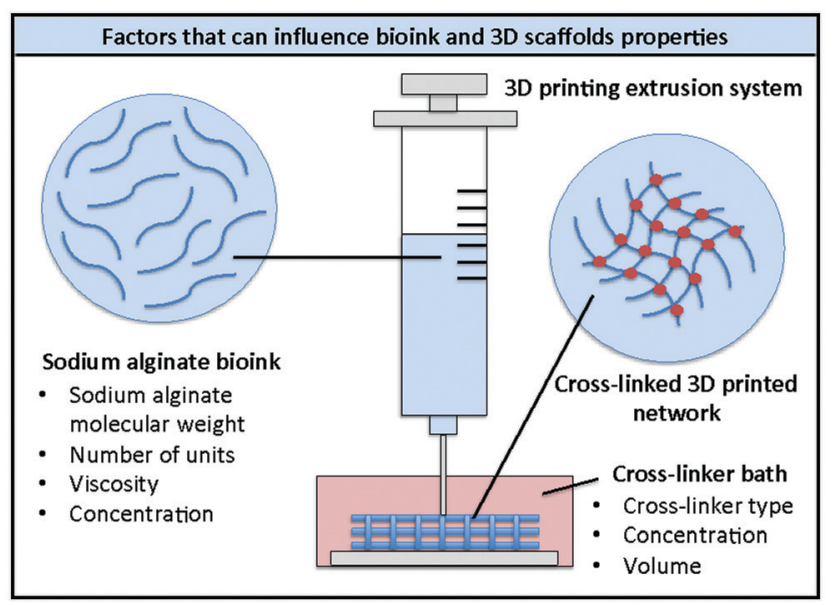

Fig. 2 Schematic representation of 3D printing of alginate bioinks and factors that can influence the properties of the bioink and the 3D printed scaffolds. 
the samples with a higher molecular weight and a higher number of $\mathrm{G}$ units.

The influence of alginate molecular weights and viscosities on bioink performance was studied by the group of Kelly, who performed a systematic study varying the ratio of alginate and cross-linker. ${ }^{42}$ They showed that the molecular weight of alginate influenced the viscosity of the resulting bioink and, consequently, the amount of cross-linker required to obtain stable 3D printed constructs. In particular, low molecular weight alginate $(28 \mathrm{kDa})$ resulted in less viscous bioinks that required 2.5 times more cross-linker than high molecular weight alginate (75 kDa).

Another crucial factor that impacts $3 \mathrm{D}$ printability and applicability of the resulting bioink is the alginate concentration. Alblas and co-workers reported alginate-based bioink formulations for gene therapy and osteogenic differentiation of embedded cells, which displayed different printabilities depending on the alginate concentration. ${ }^{43}$ The bioinks described in this study were prepared using different alginate concentrations (1.0, 2.0, 3.0 and $4.0 \% \mathrm{wt} / \mathrm{vol}$ ) and were combined with mesenchymal stem cells (MSCs). The 3D constructs were cross-linked after the printing process with a $\mathrm{CaCl}_{2}$ solution $(100 \mathrm{mM})$. The different alginate concentrations had an impact on bioink viscosity and scaffold printability. Low alginate concentrations $(<3.0 \% \mathrm{wt} / \mathrm{vol})$ gave low viscosity bioinks, which were not stable after printing. To overcome this issue, a pre-printing cross-linking step with $\mathrm{CaCl}_{2}$ (25 $\mathrm{mM}$ ) was introduced prior to extrusion. The different bioinks incorporated a plasmid containing the gene encoding the bone morphogenetic protein-2 (BMP-2), an osteogenic agent. Interestingly, they displayed differences in transfection efficiency, which were related to the polymer concentration. A low alginate concentration $(1.0 \% \mathrm{wt} / \mathrm{vol})$ resulted in $40.8 \%$ transfection efficiency, a $2.0 \% \mathrm{wt} / \mathrm{vol}$ concentration gave $35.7 \%$, a $3.0 \% \mathrm{wt} / \mathrm{vol}$ concentration gave $31.2 \%$ and $4.0 \% \mathrm{wt} / \mathrm{vol}$ resulted in only $11.8 \%$ efficiency. Clearly as the gel network becomes increasingly dense, the transfection efficiency of the plasmid is reduced, presumably as a result of its diffusion within the gel network becoming limited.

The alginate concentration in a bioink significantly affects the physical properties of the formulation, which also influences cell viability, migration and proliferation. Müller and co-workers studied this using four different alginate concentrations $(0.8 \%, 1.3 \%, 1.8 \%$ and $2.3 \% \mathrm{wt} / \mathrm{vol})$ in combination with gelatin. ${ }^{44}$ The resulting bioinks were used to 3D print MSCs scaffolds, which were cross-linked with a $2 \% \mathrm{CaCl}_{2}$ solution. The obtained constructs displayed similar porosities $(500-600 \mu \mathrm{m}$ pores), but different mechanical properties depending on the alginate concentration, with compressive moduli ranging from $1.5 \mathrm{kPa}(0.8 \% \mathrm{wt} / \mathrm{vol})$ to $14.2 \mathrm{kPa}(2.3 \% \mathrm{wt} / \mathrm{vol})$. The constructs prepared with higher alginate concentrations showed better stability over time. However, those with the lowest alginate concentration $(0.8 \% \mathrm{wt})$ showed higher cell viability after 14 days. The cells embedded in such scaffolds formed 3D interconnected networks, whereas they formed spheroids when embedded in bioinks that contained a higher alginate concentration. This suggests that, as might be expected, within a softer gel, cells are better able to exert a mechanical influence on the gel network and hence establish their own networks.

Further research on the influence of alginate concentration on cell behaviour, was carried out by the group of $\mathrm{Xu}$, who studied the effect on cell sedimentation and local concentration. ${ }^{45}$ During the printing process the cells embedded in a bioink may sediment or aggregate over time, leading to inhomogeneous cell distribution through the 3D scaffold. The researchers, therefore, tested the effect of different alginate concentrations $(0.5-4.0 \% \mathrm{wt} / \mathrm{vol})$ and different standing times (0-120 $\mathrm{min})$ on the homogeneity of 3D scaffolds of fibroblasts. They observed that the cell sedimentation velocity decreased at higher alginate concentrations. This induced a better cell distribution within the bioink over time. By contrast, the inks containing less alginate showed a nonuniform cell distribution and the formation of aggregates due to faster sedimentation. This clearly demonstrates that the polymer concentration has to be carefully chosen to achieve optimal mechanical properties for cell growth and distribution within the printed scaffold.

Another factor that influences the physical properties of the $3 \mathrm{D}$ printed constructs and their applicability is the type, concentration and volume of cross-linker. The majority of alginate hydrogels are obtained by cross-linking with calcium ions, which can come from different sources (e.g. $\mathrm{CaCl}_{2}, \mathrm{CaCO}_{3}$, $\left.\mathrm{CaSO}_{4}\right) .{ }^{46} \mathrm{CaCl}_{2}$ is a water-soluble salt; therefore, when an aqueous solution of $\mathrm{CaCl}_{2}$ is combined with sodium alginate, the $\mathrm{Ca}^{2+}$ ions are immediately available to form ionic inter-chain bridges between the polymer chains. ${ }^{17}$ In this case, gelation happens very quickly and the resulting gels can be inhomogeneous. By contrast, $\mathrm{CaCO}_{3}$ and $\mathrm{CaSO}_{4}$ are not water soluble, but can release calcium ions in an acidic aqueous environment. This process happens more slowly and, for this reason, the hydrogels obtained using these salts as calcium sources, are more homogeneous. ${ }^{46}$

The effect of different calcium ion sources $\left(\mathrm{CaCl}_{2}, \mathrm{CaCO}_{3}\right.$ and $\mathrm{CaSO}_{4}$ ) on the printability window of different molecular weight alginates was studied by Kelly and co-workers, who performed a systematic study varying the ratio of alginate and cross-linker. ${ }^{42}$ They demonstrated that the source of $\mathrm{Ca}^{2+}$ ions has an impact on the mechanical properties of the constructs. The 3D printed shapes cross-linked with $\mathrm{CaSO}_{4}$ were stiffer than those formed using the other two cross-linkers. This difference in mechanical properties was explained considering the different solubility in water of the different cross-linkers and the rates of gelation as described above. The lower solubility of $\mathrm{CaSO}_{4}$ results in slower, more uniform gelation, which improves the mechanical properties of the resulting gel. Interestingly, the stiffness of the printed cylindrical constructs was spatially controlled from the core to the periphery to direct the differentiation of encapsulated MSCs. This was achieved by printing gradient constructs, where the core of the construct was printed using the soft bioink and the external part was printed with the stiff bioink. Stiffer regions preferentially supported osteogenesis, whereas softer regions were more suitable for adipogenesis.

Chen and co-workers described the effect of gelation time and cross-linker volume on the mechanical properties of the 
resulting 3D printed alginate scaffolds. ${ }^{47}$ This study was performed by varying the gelation time between 0 and 24 hours, using different volumes of a $50 \mathrm{mM} \mathrm{CaCl}_{2}$ solution (1-5 mL). The results showed that a larger volume of cross-linking agent $(3 \mathrm{~mL}$ vs. $1 \mathrm{~mL}$ ) induced better mechanical stability immediately after printing. However, a further volume increase $(5 \mathrm{~mL})$ did not significantly improve the elastic modulus of the constructs after 24 hours from printing. Further studies on the effect of different cross-linker concentrations on the mechanical properties of the bioprinted structures were carried out by the group of Shu. ${ }^{48}$ The gels $(8.0 \% \mathrm{wt} / \mathrm{vol}$ alginate) were pre-cross-linked for $10 \mathrm{~min}$ with different $\mathrm{CaCl}_{2}$ concentrations $(50-300 \mathrm{mM})$ and then treated with a $60 \mathrm{mM}$ solution of $\mathrm{BaCl}_{2}$. The elastic modulus $\left(G^{\prime}\right)$ increased progressively with increasing concentrations of $\mathrm{CaCl}_{2}$. In particular, the $G^{\prime}$ of the gels cross-linked with a $50 \mathrm{mM}$ solution of $\mathrm{CaCl}_{2}$ was around $5.2 \mathrm{kPa}$. This increased to $c a$. 20-21 kPa when exposed to 100 or $200 \mathrm{mM} \mathrm{CaCl}_{2}$ and to $c a .28 \mathrm{kPa}$ when the cross-linker concentration was $300 \mathrm{mM}^{48}$ The second cross-linking step was decisive to improve the stability of the final constructs and to create rigidity to withstand their shape during the printing process. These bioinks incorporated human glioma cells, which showed $88 \%$ viability after 11 days of culture.

All these studies demonstrate the importance of the type and concentration of both alginate and cross-linker on the properties and applicability of the derived bioinks as summarised in Fig. 2. These factors are critical not only to define the physical properties of the resulting bioink, but also the degradability of the material, which is crucial in tissue engineering applications.

Alginate degradation normally happens by the activity of the enzyme alginate lyase, which can only be found in algae, marine invertebrates and microorganisms. ${ }^{49}$ Due to the lack of such enzymes into the human body, the in vivo degradation of alginate scaffolds mainly depends on the activity of calcium chelating agents (e.g. phosphates, citrates and lactates). ${ }^{50}$ The degradation of alginate in phosphate buffer saline solution (PBS), for example, was studied by Gao and coworkers. ${ }^{51}$ They explored the swelling ratio, degradation time and the release of the model protein bovine serum albumin (BSA) from calcium alginate hydrogels prepared using different alginate concentrations $(1.25-5.0 \% \mathrm{wt} / \mathrm{vol})$. All the gels were stable in PBS for the first three days and started to degrade after this time. As might be expected, the gels prepared with the lowest alginate concentration $(1.25 \% \mathrm{wt} / \mathrm{vol})$ were degraded faster (28 days) than those obtained using higher polymer concentrations (2.5 and 5.0\% wt/vol), which were still almost intact after 56 days. The BSA release was remarkably influenced by the gel degradation rate and it was faster for the gels that were degraded more rapidly.

In general, the degradation of alginate can be quite slow and unpredictable, but it is influenced by the properties of the polymer. ${ }^{8}$ The example described above showed how alginate concentration influences this process. However, other factors should also be taken into consideration, such as molecular weight and viscosity. Hydrogels from low molecular weight and low viscosity alginate will be degraded more easily and rapidly than materials obtained from high molecular weight and high viscosity alginate. ${ }^{8,50,52}$ This was demonstrated, for example, by Mooney et al., who studied the stiffness and degradation rate of alginate hydrogels formed by combining ionically and covalently cross-linked partially oxidized ( $1 \%$ uronic acid residues), low molecular weight $(\sim 60 \mathrm{kDa})$ and high molecular weight alginates $(\sim 120 \mathrm{kDa})$. All the hydrogels were prepared using a 2.0-3.0\% wt/vol concentration of the different types of alginate in different ratios and were cross-linked with $\mathrm{CaSO}_{4}$. Hydrogels containing a higher ratio of low molecular weight alginates, showed similar elastic moduli to those obtained from high molecular weight alginates, but were degraded more rapidly as a result of a faster separation between cross-linked domains over time.

Therefore, the choice of the type of alginate not only impacts the range of suitable applications for a bioink, but also its degradability. It is vitally important to keep this in mind when designing materials for in vivo applications. In this case, it is also important to consider that the degradation rate may differ depending on the in vivo location of an implant. The group of Patsenker monitored the degradation of fluorescently-labelled alginate gels $(0.5-1.5 \% \mathrm{wt} / \mathrm{vol})$, cross-linked with calcium gluconate $(10.0 \% \mathrm{wt} / \mathrm{vol})$, in vitro and after implantation in rat hip and myocardium. ${ }^{53}$ By using viscosity-sensitive fluorescent dyes, they were able to demonstrate that denser implants prepared with a higher alginate concentration were stable for a longer time. Moreover, they observed that the half-life of the hip implants (4 days) was shorter than the half-life of the myocardium implants, which was of 6-8 days.

Different strategies can be applied to tune the degradability of a bioink, such as modification of the molecular weight distribution of alginate by application of gamma rays, ${ }^{37,52}$ or chemical modification of the polymer structure by oxidation. ${ }^{54,55}$ Studies on the degradability of oxidised alginate-based bioinks were carried out by the group of Mei, who prepared a library of thirty inks using different oxidation percentages and concentrations of alginate. ${ }^{19}$ The oxidative chemical modification was carried out using sodium periodate to give alginate dialdehyde derivatives with different percentages of oxidation. Interestingly, variations in the alginate concentration $(2,5,8,15$ and $20 \% \mathrm{wt} / \mathrm{vol})$ and percentage of oxidation $(0,5,10$ and $15 \%)$ had a remarkable influence on the viscosity of the resulting bioinks, which was higher at higher alginate concentrations and lower degrees of oxidation. The percentage of oxidation also influenced the biodegradability of the 3D printed scaffolds, which were classified as poorly degradable ( $0 \%$ oxidation), moderately degradable ( $5 \%$ oxidation) and highly degradable (10 and $15 \%$ oxidation). The viscosity and density of the obtained bioinks and the influence of these factors on printability were systematically investigated using adipose-derived stem cells (hADSCs). All the developed hydrogels showed the capability to modulate cell proliferation and spreading, without affecting the structural integrity of the 3D printed structures after 8 days. However, the gels with a higher percentage of oxidation showed higher percentages of cell proliferation after 8 days of culture (173\% for the non-oxidised gels and $232-248 \%$ for the $5-15 \%$ oxidised gels respectively). 


\section{Other fabrication technologies for alginate 3D bioprinting}

Inkjet printing technology is based on the ejection of highresolution bioink droplets through a nozzle. ${ }^{25}$ This process can be induced by a piezoelectric transducer or a heater and requires low viscosity bioinks. Although nozzle clogging can represent an issue, this system allows rapid, cost-effective, high-resolution bioprinting of highly reproducible microdroplets or microcapsules by direct deposition of sodium alginate droplets into a cross-linker solution. ${ }^{56}$ The size and the shape of the printed droplets can be controlled by varying the cross-linker concentration and viscosity as well as the printing parameters. For example, the group of Štěpánek described the preparation of alginate microbeads $(0.5-1.0 \% \mathrm{wt} / \mathrm{vol})$ with a 50-70 $\mu \mathrm{m}$ diameter using a piezoelectric inkjet device. ${ }^{57}$ The droplets were released into magnetically stirred $\mathrm{CaCl}_{2}$ solutions $(2.0 \% \mathrm{wt} / \mathrm{vol})$ with variable viscosities $(1-100 \mathrm{mPa})$, obtained by addition of different glycerol concentrations $(0-82 \% \mathrm{wt} / \mathrm{vol})$. The droplet size was controlled by modifying the voltage applied to drive the piezoelectric print-head. Interestingly, the cross-linker viscosity remarkably influenced the shape of the resulting particles. Low viscosity cross-linker solutions gave elongated microbeads, which became spherical or flattened for medium and high viscosity solutions respectively reflecting the more heavily crosslinked structures.

The influence of cross-linker concentration on the size of 3D printed microspheres was studied by O'Leary and co-workers, who prepared alginate microcapsules $(0.5 \% \mathrm{wt} / \mathrm{vol})$ for the release of the model drug dextran-fluorescein isothiocyanate (dextran-FITC) using an inkjet piezoelectric printing system. ${ }^{58}$ The concentration of the $\mathrm{CaCl}_{2}$ cross-linker bath was varied between 1.0 and $5.0 \% \mathrm{wt} / \mathrm{vol}$. The microspheres obtained from the lowest cross-linker concentration $(1.0 \% \mathrm{wt} / \mathrm{vol}) \mathrm{had}$ a larger diameter $($ c.a. $38 \mu \mathrm{m})$ than the diameter of the particles obtained at the highest $\mathrm{CaCl}_{2}$ concentration (c.a.12 $\mu \mathrm{m}$ ), which were also more spherical and more consistent in size.

The influence of the type of inkjet technology on the resulting 3D printed alginate microbeads was studied by Schubert's group, who compared two different inkjet systems: a drop-on-demand and a continuous ink release technology. ${ }^{59}$ Both methods allowed the preparation of highly reproducible sub-nanolitre droplets, but displayed very significant differences in terms of the sizes of the generated beads. This comparative study was carried out using a $1.0 \% \mathrm{wt} / \mathrm{vol}$ alginate concentration loaded with the dye brilliant blue $\mathrm{G}$ to provide contrast, which was added dropwise to a $15.0 \%$ $\mathrm{wt} / \mathrm{vol} \mathrm{CaCl}_{2}$ bath. The beads obtained using the drop-on-demand printing technology showed a tear-drop shape with an average diameter of $48 \mu \mathrm{m}$. By contrast, the continuous inkjet system produced much larger beads with an average diameter of $248 \mu \mathrm{m}$.

Another bioprinting technology that can be applied to alginate-based bioinks is microfluidic bioprinting, which is based on the precise flow of small amounts of fluids $\left(10^{-9}\right.$ to $10^{-18} \mathrm{~L}$ volumes) through microchannels. ${ }^{26}$ This technique allows the fabrication of high resolution 3D printed microfibers or microbeads with efficient control of both morphology and dimensions. ${ }^{26}$ Noteworthy applications of microfluidic technology include the production of 3D printed vascular structures and cellular organoids..$^{27,60,61}$
As described above for the inkjet technique, in this case, the concentration of alginate and of the cross-linker once again has a remarkable effect on the features of the bioprinted structures. Aguilera and co-workers studied the effects of these two factors on the mechanical properties of calcium alginate fibres prepared using a microfluidic device. ${ }^{62}$ The fibres were obtained by introducing a $\mathrm{CaCl}_{2}$ solution $(0.5-2.5 \%)$ and an alginate solution $(1.25-2.5 \% \mathrm{wt} / \mathrm{vol})$ into a microdevice. The flow rate was controlled by two digital syringe pumps that created a vertical laminar flow, which pushed downward the calcium alginate fibres that were finally extruded into a $\mathrm{CaCl}_{2}$ bath. The isolated fibres had a diameter of $300-550 \mu \mathrm{m}$ and displayed better tensile strength and elasticity at higher alginate and cross-linker concentrations.

Juncker and co-workers studied the influence of printing parameters, such as flow rate, on fibre size and morphology. ${ }^{63}$ The 3D printed microfibers $(70-90 \mu \mathrm{m}$ diameter) were prepared using a 1.0 or $2.0 \% \mathrm{wt} / \mathrm{vol}$ alginate concentration, which was combined with a $2.0 \% \mathrm{wt} / \mathrm{vol} \mathrm{CaCl}_{2}$ solution before extrusion. The flow rate remarkably influenced the shape of the fibers and, to avoid curling or bulging, an optimal flow rate of 0.25$0.5 \mu \mathrm{L} \mathrm{min}{ }^{-1}$ had to be applied. The rigidity of the 3D printed constructs largely depended on the alginate concentration and, as expected, it was higher when the highest concentration $(2.0 \% \mathrm{wt} / \mathrm{vol})$ was used.

The use of microfluidic bioprinting to fabricate alginate microspheroidal organoids was explored, amongst others, by the group of He. ${ }^{64}$ The researchers prepared small spheroids with average diameters of $1200-1400 \mu \mathrm{m}$ by extruding a cellladen alginate solution $(2.0 \% \mathrm{wt} / \mathrm{vol})$ into a $\mathrm{CaCl}_{2}$ bath $(2.0 \%$ $w t / v o l)$. During extrusion, a gentle airflow was applied under spinning, which modified the droplet microarchitecture to obtain specific patterns such as a spiral, a rose or a saddle. This technique was applied to obtain a human multicellular organoid of spirally vascularised ossification.

All these 3D bioprinting techniques require bioinks with a suitable viscosity and shear-thinning behaviour. The viscosity of alginate bioinks can be easily tuned by modifying the molecular weight and the concentration of the polymer, thus making it easily adaptable. ${ }^{8}$ Moreover, differently from other polymers (e.g. thermally triggered polymer gelators), alginate allows cell encapsulation before printing and ensures cell survival during and after printing. ${ }^{25}$ All these properties make this material very versatile and suitable for use with different 3D printing technologies.

\section{Applications of alginate-based bioinks}

Due to their versatility and the possibility to tailor their mechanical properties as described above, alginate bioinks can be used to print a wide variety of cell types, including stem cells, fibroblasts, neurons and hepatocytes. Given the ease by which alginate systems can be fabricated, they have often been combined with other additives in order to have an impact on cell growth and behaviour. 


\section{Mesenchymal stem cells, bone and cartilage scaffolds}

Alginate bioinks have been proven to support mesenchymal stem cells growth and differentiation, with better results when prepared in combination with particles such as graphene oxide or hydroxyapatite. Lee and co-workers reported an alginate bioink formulation prepared with a $3 \% \mathrm{wt} / \mathrm{vol}$ alginate concentration, which could be used to print 3D MSCs scaffolds. ${ }^{65}$ The addition of graphene oxide $\left(0.05-1.0 \mathrm{mg} \mathrm{mL} \mathrm{mL}^{-1}\right)$ significantly enhanced the capability of the cells to undergo osteogenic differentiation, probably due to increased mechanical stiffness of the gels. The authors tried to explain this osteoinductive behaviour, by suggesting that the graphene oxide could help to support the scaffold integrity and provide mechanical cues to MSCs for osteogenesis. The best results in terms of printability and stability of the 3D printed structures were obtained with the lowest graphene oxide concentration $\left(0.05 \mathrm{mg} \mathrm{mL}^{-1}\right)$. This bioink formulation also showed the highest calcium deposition, alkaline phosphatase activity and the highest expression of osteogenic markers, and was therefore considered the most suitable for bone tissue engineering.

The group of Gümüşderelioğlu studied the applicability of alginate-based bioinks to print bone tissue from a preosteoblast mouse cell line. ${ }^{66}$ The alginate bioinks employed in this study $(3.0 \% \mathrm{wt} / \mathrm{vol})$ were prepared by internal gelation using $\mathrm{Ca}_{2} \mathrm{SO}_{4}(1.0 \% \mathrm{wt} / \mathrm{vol})$ to guarantee the formation of homogeneous gels. This procedure was followed by external gelation with $\mathrm{CaCl}_{2}(2.0 \% \mathrm{wt} / \mathrm{vol})$ for $15 \mathrm{~min}$. This method allowed a uniform distribution of ions throughout the system, providing the gel with structural homogeneity. Some of the gels reported in this study incorporated hydroxyapatite particles (20 $\mathrm{mg} \mathrm{mL}^{-1}$ ), which have been proven to increase cell attachment and lead to osteogenic differentiation from osteogenic progenitor cells. ${ }^{67,68}$ The hydroxyapatite particles were mixed homogeneously with the bioink-cell mixture during the manufacturing process. This enabled a good cell-particle interaction; moreover, the presence of hydroxyapatite further increased the viability and proliferation of the pre-osteoblast cells.

The preparation of $3 \mathrm{D}$ printed scaffolds for bone tissue engineering was also explored by the group of Grandfield, who created an osteoblast in vitro model, by printing an alginate bioink with the ExCeL technique (combining Extrusion printing on Cellulose scaffolds with Lamination). ${ }^{69}$ This technique uses chromatography paper impregnated with the crosslinker $\left(\mathrm{CaCl}_{2}\right)$ as a support for the 3D printed scaffolds. Firstly, the paper was prepared by printing a $\mathrm{CaCl}_{2}$ solution $(0.1$ or $1 \mathrm{M})$ onto it followed by drying overnight. Then, the alginate bioinks (2.0 or $3.0 \% \mathrm{wt}$ ) containing osteoblasts were printed on the paper and became immediately cross-linked when in contact with it. Interestingly, the different cross-linker and alginate concentrations yielded two gels with different Young's moduli, which induced different cell behaviour. The stiffer gels were more compatible with the formation of the osteocyte-like cells from the osteoblasts, with good overall cell viability.

Kelly and co-workers explored the applicability of alginate bioinks to support the growth of MSCs and their differentiation into chondrocytes. ${ }^{70}$ The bioinks were prepared using a $2.45 \%$ wt/vol concentration of alginate in Dulbecco's modified Eagle's Medium (DMEM) and porcine articular cartilage extracellular matrix (cECM) in a 0.2 or $0.4 \%$ wt/vol concentration. A precrosslinking step, by addition of a $0.018 \mathrm{M}$ solution of $\mathrm{CaCl}_{2}$, was initially performed to improve the viscosity of the formulation, which also showed shear-thinning and thixotropic behaviour. The bioinks were then fully cross-linked after printing in a $0.06 \mathrm{M} \mathrm{CaCl}_{2}$ bath for 20 minutes. The encapsulated cells displayed high viability $(>70 \%)$ in all of the bioinks 24 hours after printing and the obtained constructs were stable for over 42 days of culture. All the prepared bioinks induced chondrogenesis, however those containing the highest cECM concentration $(0.4 \%)$ were found to be more chondro-inductive than the others. Since the compressive stiffness of the bioinks was lower than that of native articular cartilage, the cECM bioinks were subsequently reinforced with a supporting polycaprolactone (PCL) framework, resulting in hybrid constructs with biomimetic mechanical properties.

\section{Fibroblast and vascular scaffolds}

A number of alginate bioinks were used to prepare fibroblast constructs. Park and co-workers, for example, explored the effect of different concentrations of low and high-molecular weight alginate bioinks on fibroblast growth. ${ }^{39}$ The best results in terms of processability and shapes were obtained using a $3.0 \%$ wt bioink composed of a $1: 2$ ratio of low- and high-molecular weight alginate (respectively 143 and $350 \mathrm{kDa}$ ). These formulations were successfully used to print and grow fibroblasts, which displayed good viability in the $3 \mathrm{D}$ printed scaffolds after seven days of culture. Further studies were performed by the group of Chrisey, who optimised the conditions to fabricate $3 \mathrm{D}$ alginate vascular constructs by laser printing. ${ }^{71}$ The inks containing alginate alone $(8.0 \% \mathrm{wt} / \mathrm{vol})$ or in combination with fibroblasts $(2.0 \% \mathrm{wt} / \mathrm{vol}$ alginate) were laser-printed layer by layer into straight or Y-shaped tubes and cross-linked using respectively $2.0 \%$ or $1.0 \% \mathrm{wt} / \mathrm{vol} \mathrm{CaCl}_{2}$. In both cases, the post-printing cellviability immediately after printing and after incubation for 24 hours was above $60 \%$.

The group of Yeong also used alginate-based gels (6.0\% wt/vol) to fabricate vascular-like tubular structures using a multinozzle extrusion-based technique. ${ }^{72}$ This method was based on the concurrent deposition of cross-linking agent $\left(\mathrm{CaCl}_{2}\right.$ $500 \mathrm{mM}$ ) into concentric tubular walls during each layer of deposition. Alginate was selected as the model material to demonstrate the feasibility of this versatile and simple method. Further studies on the formation of vascular constructs were undertaken by $\mathrm{He}$ and co-workers, who developed novel 3D-printed alginate structures by extrusion. ${ }^{61}$ The alginate bioinks (2.0-4.0\% wt/vol) were loaded with fibroblasts and smooth muscle cells and the $3 \mathrm{D}$ printed structures were cross-linked with $\mathrm{CaCl}_{2}$ 4.0\%. The vascular structures were obtained by extruding and printing along a rotated rod template. Endothelial cells were then seeded into the inner wall. The most successful formulation used in this study, contained a $4.0 \% \mathrm{wt} / \mathrm{vol}$ alginate concentration. The fibroblasts encapsulated in the structures showed over 90\% survival after 1 week. 
It is interesting to note here that all vascular constructs discussed above were prepared using higher alginate concentrations compared to 3D scaffolds of MSCs. This is probably because these types of structures are often more complex than others in terms of shape and number of 3D printed layers and, therefore, require a higher stability and rigidity, which can be achieved using higher polymer concentrations.

\section{Neural scaffolds}

Alginate-based bioinks were also found to be suitable with nerve tissue engineering. The group of Diáz-Diáz explored this in detail in a recent review, where they discussed the applications of alginate hydrogels as scaffolds and delivery systems to repair the damaged spinal cord. ${ }^{73}$ When applying alginate bioinks for nerve tissue engineering, very low-concentration alginate hydrogels $(0.2-1.0 \% \mathrm{wt} / \mathrm{vol})$ were more favourable to keep cell viability and function. ${ }^{35,74}$ However, low alginate concentration also leads to problems with poor printability and stability of the $3 \mathrm{D}$ printed constructs. ${ }^{75,76}$ The group of Chen tried to overcome these problems by printing low concentration alginate bioinks incorporating Schwann cells on pre-formed sacrificial gelatin scaffolds. ${ }^{77}$ The bioinks were prepared using 0.5 , 1.5 and $3.0 \%$ wt alginate concentrations and displayed differences in their swelling and degradation profiles. The scaffolds were fabricated by an indirect bioprinting process, which involved different steps (Fig. 3). Sacrificial gelatin scaffolds (50.0\% wt/vol) were initially prepared (Fig. 3a) and subsequently impregnated with the alginate solutions (Fig. 3b). These were cross-linked with $\mathrm{CaCl}_{2}(50 \mathrm{mM})$ added on top of the frameworks. The scaffolds were then refrigerated for 18 hours and placed in an incubator with $5.0 \% \mathrm{CO}_{2}$ at $37{ }^{\circ} \mathrm{C}$ to melt, and hence remove the gelatin (Fig. 3c). As might be expected, the best results in terms of stability of the resulting 3D printed constructs were obtained with the highest alginate concentrations (1.5 and 3.0\% wt/vol, Fig. $3 \mathrm{~b}$ and $\mathrm{c}$ ). However, the scaffolds prepared with the lowest alginate concentration showed the best results in terms of cell growth, migration and proliferation.

A similar approach, in this case described as 'FRESH' (freeform reversible embedding of suspended hydrogels), was adopted by the group of Hermanson, who reported a moderately low concentration alginate bioink $(2.0 \% \mathrm{wt} / \mathrm{vol})$ for $3 \mathrm{D}$ bioprinting of human neuroblastoma cells. ${ }^{78} \mathrm{~A}$ gelatin slurry was used for physical support during printing and $\mathrm{CaCl}_{2}$ $(100 \mathrm{mM})$ was used as an ionic cross-linker for the alginate chains. The gelatin sacrificial template was then removed by incubating the scaffolds at $37{ }^{\circ} \mathrm{C}$. The cells embedded in the scaffolds showed good viability and proliferation after 7 days from printing.

It is important to note here how lower alginate concentrations are more favourable to grow neural tissues. However, since these often lead to poorly printable and stable 3D printed scaffolds, the use of sacrificial gelatin templates seems to be an effective solution. Exploring bioinks with different alginate and cross-linker concentrations or prepared in combination with other additives is probably an area that it would be worth further investigating to identify optimal conditions for neural tissue growth.

\section{Hepatocyte scaffolds}

Alginate-based bioinks were also employed to 3D print hepatocyte constructs. The group of Shu, for example, explored the conditions to bioprint hepatocyte-like cells without affecting their biological function and pluripotency. ${ }^{79}$ The printing process was performed by extrusion using a $1.5 \% \mathrm{wt} / \mathrm{vol}$ concentration of
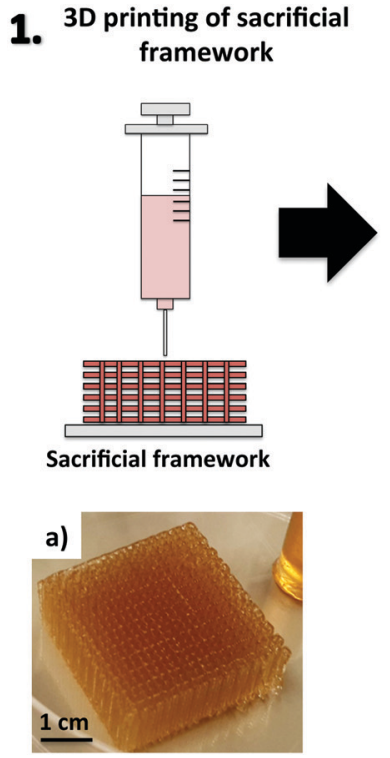
2. Addition of alginate bioink and
subsequent cross-linking
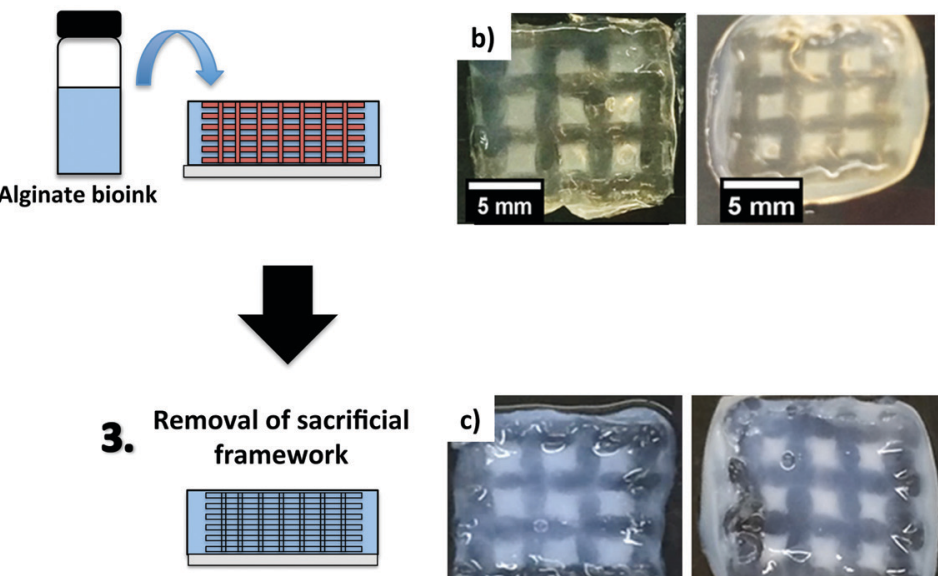

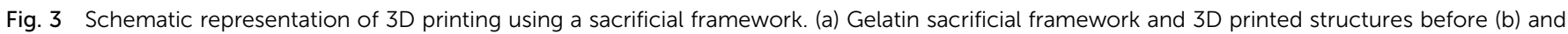

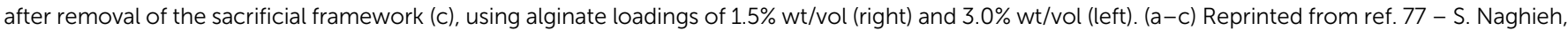

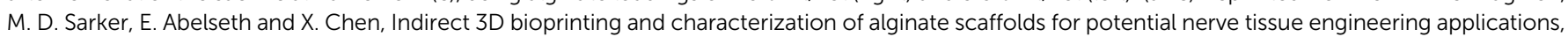
J. Mech. Behav. Biomed., 2019, 93, 183-193. Copyright (2019), with permission from Elsevier. 
sodium alginate, which was previously combined with the cells and cross-linked with $\mathrm{CaCl}_{2} 60 \mathrm{mM}$. This was followed by a second postprinting cross-linking step using $\mathrm{BaCl}_{2}(55 \mathrm{mM})$. Interestingly, the nozzle length affected the post-printing viability of the cells. The best results were obtained when shorter nozzles were used.

All of these studies demonstrate the versatility of alginate bioinks, which can display different mechanical properties depending on the alginate concentration and the cross-linker. This allows tailoring of the resulting bioink to create a suitable environment for different cell types. In particular, we note the approaches to bioprinting which make use of temporal control, such as sacrificial scaffolds or later additional cross-linking steps - in this way, the properties of a scaffold can be modulated at different points in a 3D printing and cell growth experiment, providing a degree of fine control.

\section{Multicomponent bioinks obtained combining alginate with other polysaccharides}

As discussed above, alginate alone can be applied to obtain 3D printed constructs of a wide range of cell types. However, the majority of alginate-based bioinks reported in the literature are formulated by combining alginate with other polymers. This allows the rheology and the stability of the resulting bioinks to be improved, adapting their properties to specific applications.

We herein report the main types of bioink formulations prepared by combining alginate with other polysaccharides (i.e. agarose, cellulose, methylcellulose, hyaluronic acid and gellan gum; Fig. 4). We aim to highlight here how such formulations are prepared and how the properties of the alginate-based bioinks are improved by the addition of the second polysaccharide component. We note that the systems including sacrificial gelatin described in the previous section also make use of a secondary component to assist with printability, but in this case, the gelatin was removed from the hybrid material prior to the main phase of cell growth, and therefore we categorised those systems as 'alginate-only'.

\section{Bioink formulations based on alginate and agarose}

Agarose is a biopolysaccharide derived from agar, a natural product extracted from red seaweed. It is composed of basic repeating units of agarobiose, consisting of 1,3-D-galactopyranose and 3,6-anhydro- $\alpha$-L-galactopyranose (Fig. 4a). This polymer undergoes thermal cross-linking and forms hydrogels on cooling at relatively low temperatures (around $40{ }^{\circ} \mathrm{C}$ ). ${ }^{80}$ The sol-gel transition temperature depends on several factors, including the average molecular weight of agarose and its concentration in aqueous solution. Agarose hydrogels are biocompatible and have been applied in tissue engineering and drug delivery. ${ }^{81-84}$ A number of agarose-based bioinks have been reported in literature, however, despite its excellent gelation properties, this material is brittle, has a limited ability to support cell growth and, being very viscous, is not suitable with dropletbased bioprinting techniques. Another limitation is that, since it requires a heat-cool cycle to undergo gelation, it needs temperature control in the reservoir and during the printing process. These limitations can be addressed by blending it with other polymers, such as alginate, that can improve cell viability and printability. Agarose-alginate blends have been mainly reported as bioinks for the 3D printing of cartilage, neural and endothelial constructs.

Cartilage scaffolds. Agarose-based bioink formulations for 3D printed cartilaginous scaffolds were described by Kelly and co-workers, who compared the capability of four different bioinks based on agarose, alginate, gelatin methacryloyl (GelMA) and poly(ethylene glycol) methacrylate (PEGMA) to induce the in vitro development of hyaline cartilage or fibrocartilage. ${ }^{85}$ This experiment was conducted by culturing mesenchymal stem cells in the different bioinks, treated with the growth factor TGF- $\beta 3$, and by checking the markers corresponding to each cartilage type. In this case printing was carried out by extrusion. Interestingly, alginate and agarose bioinks (respectively 3.5 and 2.0\% concentration) better supported the development of hyaline-like cartilage, whereas the other two inks facilitated the development of fibrocartilaginous tissue. The alginate gel used in this study was supported by an agarose framework. The bioink was prepared by dissolving alginate in PBS and combining the resulting a)

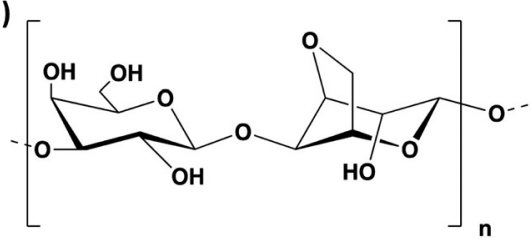

d)

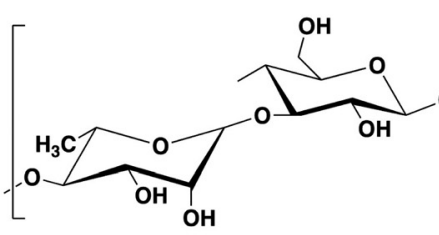

b)

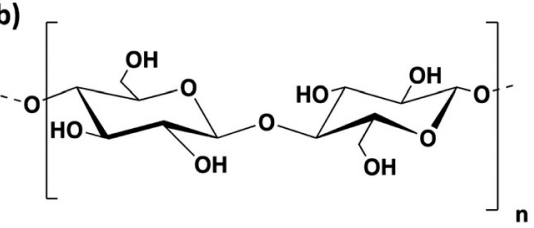

c)

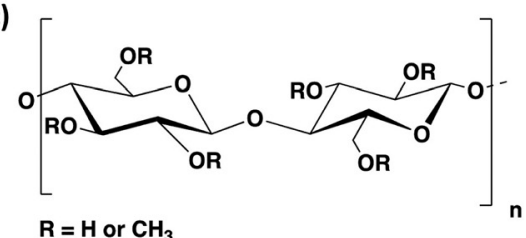

e)

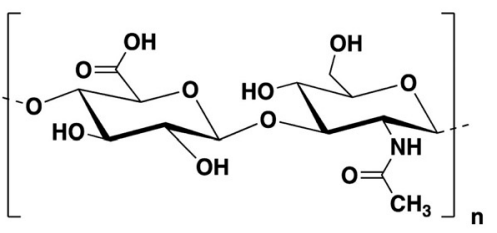

Fig. 4 Chemical structure of (a) agarose, (b) cellulose, (c) methylcellulose, (d) gellan gum and (e) hyaluronic acid. 
solution with the cells and a $60 \mathrm{mM} \mathrm{CaCl}_{2}$ solution. This was then poured into a $4.0 \mathrm{wt} \%$ agarose solution mixed with $60 \mathrm{mM} \mathrm{CaCl}_{2}$ and allowed to cross-link for $30 \mathrm{~min}$ at $37^{\circ} \mathrm{C}$.

The use of agarose-alginate composites as bioinks for the 3D printing of cartilage constructs was studied by O'Connell's group, who compared different alginate-agarose bioink formulations to the poloxamer Pluronic, which is known to have good printing properties. ${ }^{86}$ The ink composition with the best rheological properties for bioprinting (i.e. yield stress and storage modulus) was a $5.0 \% \mathrm{wt} / \mathrm{vol}$ bioink composed of alginate and agarose in a $2: 3$ ratio. This gel displayed the best printing fidelity, maintained excellent cell viability after printing (more than $95 \%$ ), and had continuous matrix production throughout the culture period.

Neural scaffolds. The 3D printing of neural constructs is another application of agarose-alginate bioinks. The group of Crook described a three-component formulation comprising agarose, alginate and carboxymethyl-chitosan, which could encapsulate human neural stem cells and support the in situ differentiation to functional neurons and supporting neuroglia. ${ }^{87}$ In the resulting bioink, alginate and agarose provided the required viscosity for printing and the structural support to the 3D printed structure after gelation, whereas the carboxymethylchitosan guaranteed cell survival within the construct. This formulation contained $5.0 \% \mathrm{wt} / \mathrm{vol}$ alginate, $1.5 \% \mathrm{wt} / \mathrm{vol}$ agarose and $5.0 \% \mathrm{wt} / \mathrm{vol}$ carboxymethyl-chitosan. Interestingly, the concentration of agarose in the ink had a remarkable influence on viscosity and the $3 \mathrm{D}$ resolution of the printed objects. Inks with lower $(0.5 \% \mathrm{wt} / \mathrm{vol})$ or higher agarose content $(2.5 \% \mathrm{wt} / \mathrm{vol})$ than the optimal concentration $(1.5 \% \mathrm{wt} / \mathrm{vol})$, did not display ideal printing viscosities and the resulting 3D structures were poorly defined. In subsequent work, the researchers used this bioink formulation to bioprint induced pluripotent stem cells (iPSCs). ${ }^{88}$ The cells were encapsulated into the polymer blend before printing and could proliferate within the 3D printed constructs and differentiate into self-organizing embryoids or neural tissues containing migrating neurons and neuroglia.

Endothelial scaffolds. More recently, Ye and co-workers reported an alginate-agarose hydrogel composite treated with platelet-rich plasma (PRP), which was used as a bioink for the $3 \mathrm{D}$ printing of human umbilical vein endothelial cells (HUVECs). ${ }^{89}$ This formulation was deposited on the internal pores of a heart-shaped polyvinyl alcohol (PVA) sacrificial scaffold, which was then removed by immersion and multiple washings in cell culture medium (DMEM) to give a flexible, hollow, micro-fluid channel network structure. The alginateagarose blend was prepared in phosphate buffer saline (PBS) solution using $2.0 \% \mathrm{wt} / \mathrm{vol}$ alginate with different concentrations of low-melting point agarose $(0.6 \%, 1.2 \%, 1.8 \%$ and $2.4 \% \mathrm{wt} / \mathrm{vol})$. These were combined with PRP and cross-linked with $2.0 \% \mathrm{CaCl}_{2}$. The agarose concentration was crucial to determine the viscosity of the resulting bioinks, optimal printability and stability of the 3D printed shapes. In this case, the viscosity of the inks containing the highest agarose concentration gave the best results in terms of processability and stability of the printed object. By contrast, the inks prepared with lower agarose concentrations were too liquid to allow effective ink deposition on the sacrificial PVA template.

\section{Bioink formulations based on alginate and cellulose}

Cellulose is a natural polysaccharide composed of $\beta(1-4)$-linked D-glucose units (Fig. 4b). It can be obtained from plants or bacterial biosynthesis. Cellulosic extracts with one dimension in the nanometre range are also known as nanocellulosic materials and can be classified as nanofibrillated cellulose (NFC) and cellulose nanocrystals (CNCs). ${ }^{90}$ NFCs are long cellulose fibrils containing amorphous and crystalline regions, extracted from plants by a combination of mechanical and chemical treatments. Such fibres can form highly entangled networks in aqueous media with high viscosity at very low concentrations (below 1\% wt/vol). CNCs, on the other hand, are crystalline rod-like particles, mainly extracted by acid hydrolysis. This process disrupts the amorphous fibre domains, allowing the isolation of crystal nanoparticles with well-defined shapes. Being a natural, abundant, environmentally friendly, cost-effective, biodegradable and biocompatible resource, hydrogels from cellulose have been widely employed in tissue engineering, drug delivery and wound healing. ${ }^{91-96}$ A wide variety of cellulose bioinks has been reported in the literature, especially in the last few years. ${ }^{97-101}$ The combination of this polysaccharide with alginate yielded a number of different formulations, which were mainly applied for the 3D printing of cartilage scaffolds.

Cartilage scaffolds. The group of Gatenholm is one of the leading groups in the field and has studied in detail the properties and applications of nanocellulose-alginate bioinks. In 2015, they reported various NFC-alginate blends composed of different ratios of the two polymers and studied the printing parameters and shape fidelity. ${ }^{102}$ The polymer mixtures were prepared using a $2.5 \% \mathrm{wt} / \mathrm{vol}$ NFC dispersion and $2.5 \% \mathrm{wt} / \mathrm{vol}$ alginate solution combined in different ratios: $90: 10,80: 20$, 70:30 and 60:40 and cross-linked with a $90 \mathrm{mM} \mathrm{CaCl}_{2}$ solution. All the inks displayed similar viscosity and shear thinning behaviour. Interestingly, before cross-linking, the properties of the NFC were dominating, allowing shear thinning behaviour, high printing resolution and shape fidelity. After cross-linking, the alginate properties dominated, with higher elastic modulus values for the gels that contained a higher amount of alginate. These bioinks were used to bioprint chondrocyte scaffolds, which showed good cell viability after 7 days of culture. In subsequent work, they studied the applicability of a commercially available NFC-alginate bioink ('CELLINK') to bioprint complex cell-laden cartilage constructs (e.g. a human ear-shaped scaffold) with controlled cell density and porosity. ${ }^{18}$ The bioink used in this project contained $2.0 \%$ $\mathrm{wt} / \mathrm{vol} \mathrm{NFC}$ and $0.5 \% \mathrm{wt} / \mathrm{vol}$ alginate and it was cross-linked after printing in a $100 \mathrm{mM} \mathrm{CaCl}_{2}$ bath. The ear-shaped constructs showed high shape stability after printing and were used to grow auricular and nasal chondrocytes with good cell adhesion, proliferation and maintenance of chondrogenic phenotype. Interestingly, the seeded cells underwent chondrogenesis in the bioink, with neo-synthesis and accumulation of cartilage-specific extracellular matrix around the cells. 
To improve the delivery of bioactive molecules from such formulations, these researchers subsequently developed a NFC-alginate bioink using a sulfated form of alginate. ${ }^{103}$ This chemical modification allowed the binding of growth factors and induced collagen II deposition and the proliferation of encapsulated bovine chondrocytes. These bioinks were prepared using $1.0 \% \mathrm{wt} / \mathrm{vol}$ alginate sulfate and $1.36 \% \mathrm{wt} / \mathrm{vol}$ nanocellulose, and were cross-linked after printing with a $100 \mathrm{mM}$ $\mathrm{CaCl}_{2}$ solution. The obtained formulation allowed the printing of complex 3D structures with high shape fidelity. The cell behaviour was greatly influenced by the printing conditions, with the best results in terms of preservation of cell function obtained using wide diameter conical needles.

In more recent work, the team investigated the printability of mechanically processed lipoaspirate (i.e. fat tissue removed by liposuction) containing adipose-tissue derived stem cells (ASCs), in combination with a NFC-alginate blend (Fig. 5). ${ }^{104}$ The resulting bioink (containing NFC and alginate in a $4: 1$ ratio) was studied in terms of printability and in vivo cell survival, and neovascularization of the obtained bioprinted grafts. The collected results demonstrated the applicability of this bioink to print complex and heterogeneous tissues containing various cell types including adipocytes, endothelial cells and ASCs. The 3D constructs preserved both structure and cellular composition; moreover 30 days after the scaffold was subcutaneously implanted in the neck of nude mice, evidence of vascularization was confirmed. This work is of great impact, as it clearly demonstrates the in vivo applicability and potential clinical use of such $3 \mathrm{D}$ printed constructs.

Further studies on NFC-alginate bioinks were carried out by Simonsson and co-workers, in collaboration with the group of Gatenholm. They compared the performance of such formulations to a bioink polymer blend composed of hyaluronic acid (HA) and NFC. ${ }^{105}$ The inks were used to bioprint human pluripotent stem cells. Interestingly, the NFC-alginate ink had a much better performance than the NFC-HA ink, which showed little or no proliferation of the encapsulated cells.
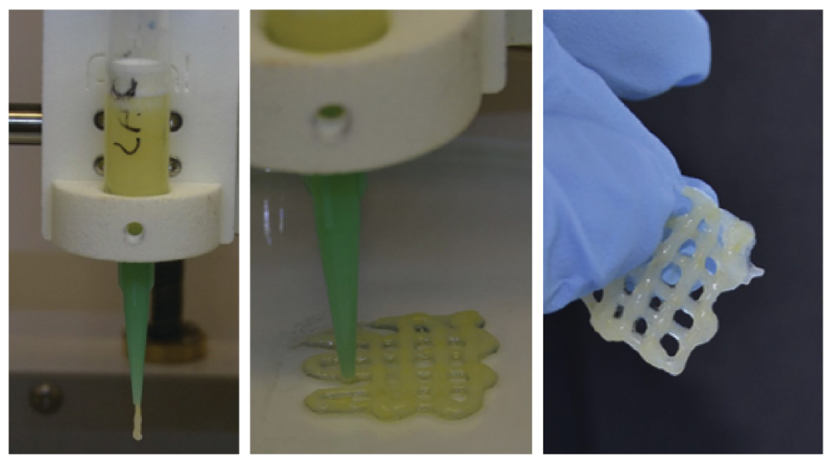

Fig. 5 3D printing of NFC-alginate blend combined with mechanically processed lipoaspirate. Reprinted from ref. 104-K. Säljö, L. S. Orrhult, P. Apelgren, K. Markstedt, L. Kölby and P. Gatenholm, Successful engraftment, vascularization, and In vivo survival of 3D-bioprinted human lipoaspirate-derived adipose tissue, Bioprinting, 2020, 17, e00065. Copyright (2020), with permission from Elsevier.
This result is quite surprising, considering that hyaluronic acid is a natural component of cartilage $\mathrm{e}^{106}$ and has been proven to encapsulate human stem cells and support their 3D growth. ${ }^{107}$ The authors hypothesized that it may have been caused by the $\mathrm{HA}$ cross-linker (hydrogen peroxide $-\mathrm{H}_{2} \mathrm{O}_{2}$ ), which may have induced phenotypic changes in the cells encapsulated into the NFC-HA gel. The best results were obtained for the NFCalginate bioink containing the two components in a $60: 40$ ratio (dry weight\%), which were cross-linked with $100 \mathrm{mM} \mathrm{CaCl}$. The 3D printed scaffolds obtained using this bioink showed good cell viability, high cell density and the formation of spherical cell clusters initially and then cartilaginous tissue after 5 weeks. The NFC-alginate constructs prepared with a $80: 20$ ratio of the two polymers, displayed a higher stability compared to the $60: 40$ bioink, but a lower cell survival rate. The live cells in this bioink, however, were evenly distributed and proliferated into elongated cell clusters. This illustrates how multicomponent systems can be easily tuned by simple formulation to achieve different outcomes.

Kolbi and co-workers explored the applicability of NFCalginate bioinks to cultivate and grow mesenchymal stem cells and chondrocytes together. ${ }^{108,109}$ In their research, they used the commercially available CELLINK formulation to bioprint 3D constructs, which were implanted into nude mice to induce the in vivo formation of viable cartilage. This study was performed by encapsulating in the ink human nasal chondrocytes in combination with MSCs. The chondrocytes displayed good viability, proliferation and cartilage-cluster formation and the MSCs induced enhanced chondrocyte proliferation.

\section{Bioink formulations based on alginate and methylcellulose}

Methylcellulose is a methyl ether of cellulose containing 27.5$31.5 \%$ methoxy groups (Fig. 4c). This polymer is widely used as a thickener and emulsifier in food and cosmetic products. ${ }^{110}$ It forms thermo-reversible hydrogels and can be applied for a variety of biological applications such as cell culture, wound healing and tissue engineering. ${ }^{111,112}$ Due to its ease of gelation and biocompatibility, it is an ideal ingredient for bioink formulations.

The properties and printability of several methylcellulosealginate bioink formulations were studied by the group of Chen, who explored the swelling, degradation rate and mechanical properties of various polymer blends in comparison to pure alginate. ${ }^{113}$ Four different formulations were analysed: pure alginate $(3.0 \% \mathrm{wt} / \mathrm{vol})$, gelatin-alginate composite $(1.0 \% \mathrm{wt} / \mathrm{vol}$ gelatin and $3.0 \% \mathrm{wt} / \mathrm{vol}$ alginate), methylcellulose-alginate composite $(1.5 \% \mathrm{wt} / \mathrm{vol}$ methylcellulose and $1.5 \% \mathrm{wt} / \mathrm{vol}$ alginate) and gelatin-methylcellulose-alginate composite $(1.0 \% \mathrm{wt} / \mathrm{vol}$ gelatin, $0.5 \% \mathrm{wt} / \mathrm{vol}$ methylcellulose and $1.5 \% \mathrm{wt} / \mathrm{vol}$ alginate). It was demonstrated that the polymer blends had a higher water absorption ability compared to pure alginate. Moreover, they displayed a higher capability to retain compressive strength over time. Interestingly, the methylcellulose-alginate bioink had the highest elastic modulus $(113.3 \mathrm{kPa})$ compared to the other formulations, which had $G^{\prime}$ values between 69.4 and $82.85 \mathrm{kPa}$ depending on the ink composition. 
A number of methylcellulose-alginate bioinks have been reported and applied to make 3D scaffolds of various cell types including, amongst others, fibroblasts, MSCs and chondrocytes.

Fibroblast scaffolds. $\mathrm{Li}$ and co-workers investigated the properties of highly robust, high loading methylcellulosealginate blends for the 3D printing of fibroblasts. ${ }^{114}$ The bioink that gave the best results was composed of $3.0 \% \mathrm{wt} / \mathrm{vol}$ alginate and $9.0 \% \mathrm{wt} / \mathrm{vol}$ methylcellulose and showed thixotropic behaviour and great extrudability. This formulation allowed the fabrication of constructs with 150 overlapping layers. The encapsulated fibroblasts displayed good cell morphology and viability after 5 days of culturing. The prepared bioinks were treated with the chelating agent trisodium citrate to remove interfacial calcium ions. A subsequent post-cross-linking process by immersion of the 3D printed constructs into a $\mathrm{CaCl}_{2}$ bath helped to build new more robust interfacial connections between layers, improving the interfacial bonding strength.

Mesenchymal stem cells, cartilage and bone scaffolds. The group of Lode reported an a methylcellulose-alginate bioink with high stability and elasticity, for the encapsulation and 3D bioprinting of MSCs. ${ }^{115}$ Once again, this ink was produced using a high total loading of $3.0 \% \mathrm{wt} / \mathrm{vol}$ alginate and $9.0 \% \mathrm{wt} / \mathrm{vol}$ methylcellulose (1:3 ratio), increasing the ink viscosity and microporosity, and tuning the rheological properties of the final ink formulation. Post-printing cross-linking was performed using a $100 \mathrm{mM} \mathrm{CaCl}_{2}$ solution. The MSCs were incorporated into the ink before 3D bioprinting and showed good viability up to 3 weeks of cultivation. The cells embedded into the $3 \mathrm{D}$ printed constructs maintained their differentiation potential over time by means of differentiation into adipocytes when the cells were incubated for 21 days with adipogenic medium.

More recently, this research group, in collaboration with Gelinsky and co-workers, performed a systematic study on the influence of different sterilization techniques (i.e. autoclave, supercritical $\mathrm{CO}_{2}$ treatment, and $\mathrm{UV}$ and $\gamma$ irradiation) on the material properties of such methylcellulose-alginate bioinks (3.0\% wt/vol alginate and $9.0 \% \mathrm{wt} / \mathrm{vol}$ methylcellulose), as well as the cellular responses. ${ }^{116}$ These bioinks were used for the bioprinting of embedded bovine chondrocytes. Cross-linking with a $100 \mathrm{mM} \mathrm{CaCl}_{2}$ solution was performed after printing. The experiments demonstrated that exposure to $\gamma$ irradiation had an impact on the polymer blend viscosity and stability after extrusion, both of which were lower compared to those of the bioinks exposed to the other treatments. Moreover, this sterilization method influenced the methylcellulose chain mobility within the gel network after alginate cross-linking with $\mathrm{Ca}^{2+}$ ions. The best results in terms of cell survival and function were displayed by the gels treated with supercritical $\mathrm{CO}_{2}$ or UV irradiation.

Methylcellulose-alginate bioinks can also be combined with other components to direct cell behaviour. Gelinsky and co-workers have investigated the effect of the incorporation of LAPONITE $^{\circledR}$, a synthetic nanosilicate clay, on the printing properties of a methylcellulose-alginate blend. ${ }^{117}$ This bioink was prepared by combining a $3.0 \% \mathrm{wt} / \mathrm{vol}$ alginate solution with LAPONITE ${ }^{\circledR}$ $(3.0 \% \mathrm{wt} / \mathrm{vol})$ and different methylcellulose concentrations
(3.0\%, $6.0 \%$ or $9.0 \% \mathrm{wt} / \mathrm{vol})$. The bioink was cross-linked with $\mathrm{CaCl}_{2}(100 \mathrm{mM})$. This blend was used to create 3D scaffolds incorporating MSCs with high printing fidelity and showing cell viability for more than 21 days. Since LAPONITE ${ }^{\circledR}$ is known for its drug delivery properties, to prove the applicability of this hydrogel composite as a drug delivery system, the bioink was loaded with two model proteins (bovine serum albumin (BSA) and vascular endothelial growth factor VEGF)). The release of these proteins from the hydrogel in the cell medium was significantly improved in the presence of LAPONITE ${ }^{\circledR}$ compared to the same inks without LAPONITE ${ }^{\mathbb{R}}$, although a high amount was retained. To verify if the released proteins could keep their function, they incubated endothelial cells in the release medium from VEGF-laden scaffolds. The cells showed enhanced proliferation in comparison to the negative controls.

More recently, these researchers explored the effect of adding calcium phosphate cement (CPC) to a methylcellulosealginate bioink (3.0\% wt/vol alginate and 9.0\% wt/vol methylcellulose) with embedded MSCs. ${ }^{118}$ The preparation of this formulation required a certain degree of optimization in terms of printability and cell survival inside the bioinks. Once optimal conditions were identified, the system could be applied to prepare 3D osteochondral tissue grafts models.

Other applications. The group of Gelinsky has published a remarkable number of scientific articles on 3D bioprinting of methylcellulose-alginate composites. In one of the reported studies, using a highly viscous blend $(3.0 \% \mathrm{wt} / \mathrm{vol}$ alginate and 9.0\% wt/vol methylcellulose), cross-linked with $\mathrm{SrCl}_{2}$, to obtain $3 \mathrm{D}$ printed constructs of viable and functional pancreatic islets from rats. ${ }^{119}$ The printed cells kept their function and morphology and it was possible to recognize $\alpha$ and $\beta$ pancreatic cells within the islets.

Further research described the properties of a more complex formulation composed of methylcellulose (3.0\% wt/vol), alginate $(2.8 \% \mathrm{wt} / \mathrm{vol})$ and agarose $(0.9 \% \mathrm{wt} / \mathrm{vol})$, which was used for the incorporation and bioprinting of plant cells. ${ }^{120}$ The bioink containing a living cell culture of basil was printed by extrusion using different ratios of the three polymers. They observed that the methylcellulose concentration was crucial to ensure good printability and maintain shape fidelity. Bioinks containing lower methylcellulose content, displayed low viscosity, loss in 3D printing definition and loss of stability of the 3D printed constructs and in their capability to retain the shape. Cell survival on the printed structures was confirmed by live/dead staining, microscopy and metabolic measurements.

\section{Bioink formulations based on alginate and gellan gum}

Gellan gum is a biopolymer of bacterial origin, produced by the bacterium Sphingomonas Elodea. This polysaccharide is composed of tetrasaccharidic repeating units consisting of two residues of D-glucose, one residue of L-rhamnose and one residue of D-glucuronic acid (Fig. 4d). Hydrogels of gellan gum are obtained by thermal trigger or/and by cross-linking with divalent cations, such as $\mathrm{Ca}^{2+}, \mathrm{Ba}^{2+}$ and $\mathrm{Sr}^{2+} \cdot{ }^{80,121,122}$ Such hydrogels have been widely applied in the food industry, ${ }^{123,124}$ however, more recently they have also been shown to be suitable 
for drug delivery and tissue engineering applications. ${ }^{121,122,125}$ Being biocompatible, gellan gum is an ideal component to improve the mechanical properties of alginate bioink formulations.

Mesenchymal stem cells, cartilage and bone scaffolds. Zenobi-Wong and co-workers reported a gellan gum-alginate bioink with shear-thinning behaviour and optimal printability, which was cross-linked by delivering cations by co-extrusion of a cation-loaded transient support polymer $30 \%$ pluronic in $\mathrm{NaCl}$ and $\left.\mathrm{SrCl}_{2}\right) .{ }^{126}$ This ink was prepared by combining $3.0 \% \mathrm{wt} / \mathrm{vol}$ gellan gum with $2.0 \% \mathrm{wt} / \mathrm{vol}$ alginate and it was used to produce various types of grafts with different 3D shapes (i.e. human ear, nose, meniscus and vertebral disk). These grafts were treated with cartilage extracellular matrix particles (BioCartilage), and it was shown that this bioink could support proliferation of chondrocytes and the deposition of cartilage matrix proteins. This was observed to a higher extent when the bioinks were treated with the chondrogenic protein transforming growth factor $\beta$ (TGF- $\beta$ ).

In subsequent work, these researchers described the effect of incorporating cationic-modified silica nanoparticles into an anionic polymer blend composed of alginate and gellan gum (3.0\% and $3.5 \% \mathrm{wt} / \mathrm{vol}$, respectively). ${ }^{127}$ This combination resulted in a significant increase in zero-shear viscosity (1062\%) and storage modulus (486\%). The presence of the silica nanoparticles therefore allowed an increase of stability and shape fidelity of the 3D printed constructs, which did not collapse during printing. Interestingly, the size of the nanoparticles had to be $<100 \mathrm{~nm}$ to guarantee such mechanical enhancement and they also reduced shrinking and swelling of the obtained constructs. In general terms, the impact of nanoparticles on the mechanical performance of soft materials is quite well-known. ${ }^{128-131}$ More importantly, the incorporated nanoparticles did not affect the bioink biocompatibility with the growth of encapsulated chondrocytes, which displayed high cell viability $(>90 \%)$ and matrix production.

The use of gellan gum-alginate bioinks for 3D printing and in situ differentiation of MSCs was investigated by the group of Gelinsky. Highly stable bioinks with good processability and enhanced mechanical properties were obtained by combining $3.0 \% \mathrm{wt} / \mathrm{vol}$ gellan gum with $2.0 \% \mathrm{wt} / \mathrm{vol}$ alginate, cross-linked with $1 \mathrm{M} \mathrm{CaCl}_{2} \cdot{ }^{132}$ Compared to $3 \mathrm{D}$ printed scaffolds of pure alginate, the presence of gellan gum improved mechanical strength, shape fidelity and decreased the swelling in cell culture medium. The prepared bionks were compatible with the growth of MSCs and supported osteogenic differentiation. However, after two weeks of culture the number of viable cells decreased. Long-term cell culture may be improved using other divalent cations (e.g. $\mathrm{Sr}^{2+}$ ), which form more stable cross-linked hydrogels and are therefore more suitable for long term studies. ${ }^{133,134}$ Another option could be coating with a peptide that encourages cell proliferation, such as the tripeptide Arg-Gly-Asp (RGD), which has been proven to improve cell adhesion, spreading and proliferation. ${ }^{22,135-137}$

The researchers subsequently reported a biphasic scaffold incorporating VEGF, obtained by combining a gellan-gum/ alginate bioink hydrogel composite with an oil-based calcium

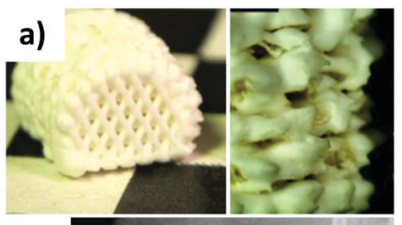

b)

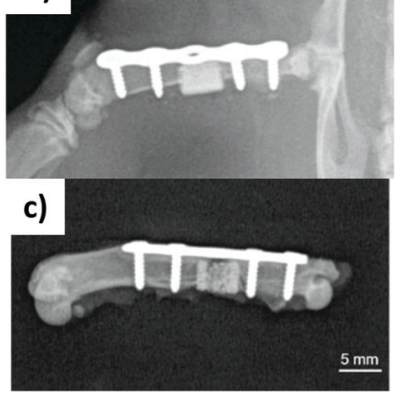

Fig. 6 Gellan gum/alginate/CPC 3D printed 75 scaffold (a) immediately after printing, (b) after implantation into rat femur (post-operative X-ray, and (c) after explantation after 12 weeks. Adapted from ref. 138 - T. Ahlfeld, F. P. Schuster, Y. Förster, M. Quade, A. R. Akkineni, C. Rentsch, S. Rammelt, M. Gelinsky and A. Lode, Adv. Healthcare Mater., 2019, 8, 1801512. Copyright 2019 WILEY-VCH Verlag GmbH \& Co. KGaA, Weinheim, with permission from Wiley.

phosphate cement (CPC - Fig. 6a). ${ }^{138}$ The CPC paste hardens in water forming nanocrystalline hydroxyapatite. The performed experiments demonstrated that the bioprinted construct was compatible with the growth of MSCs, which could differentiate towards osteoblasts. The VEGF released from the scaffold kept its function and could stimulate endothelial cell proliferation and angiogenesis in vitro. The 3D printed scaffold was implanted into a segmental bone defect in the femur diaphysis of rats (Fig. $6 \mathrm{~b}$ and c) and significantly helped reduce the defect size, by helping the formation of new bone tissue.

\section{Bioink formulations based on alginate and hyaluronic acid}

Hyaluronic acid (HA) is a linear natural polysaccharide composed of D-glucuronic acid and $N$-acetyl-D-glucosamine units linked by $\beta$ (1-3) or $\beta$ (1-4) bonds (Fig. 4e). ${ }^{139}$ This anionic glycosaminoglycan has a molecular weight ranging between 5 and $2000 \mathrm{kDa}$. Being a component of the extracellular matrix in most of the connective tissues in the body, it has the advantage of being non-toxic, non-immunogenic and non-inflammatory. ${ }^{139}$ Hydrogels of hyaluronic acid can be obtained by covalent crosslinking and they have been applied in cell culture, tissue engineering, drug delivery and wound healing. ${ }^{140-144}$ A wide variety of HA bioink formulations has been reported in the literature, ${ }^{145-148}$ some of which include hyaluronic acid-alginate blends, which were suitable for the fabrication of neural and cartilaginous scaffolds.

Neural scaffolds. Chen's group focussed on HA-alginate bioinks encapsulating Schwann cells for potential use in peripheral nerve tissue engineering. ${ }^{149}$ These formulations contained hyaluronic acid $(0.25 \% \mathrm{wt} / \mathrm{vol})$ and alginate $(2.5 \% \mathrm{wt} / \mathrm{vol})$ and were crosslinked with $\mathrm{CaCl}_{2}(100 \mathrm{mM})$. After printing the first layer, the obtained 3D construct was coated with polyethylenimine (PEI) 
to immobilise the first printed layer onto the platform. Each subsequent layer was then extruded into a $\mathrm{CaCl}_{2}$ bath to cross-link the alginate. Polyvinyl alcohol (PVA) and PEI were also added to improve the shape fidelity and the stability of the resulting scaffolds. The resulting 3D printed constructs displayed high structural integrity over time, whilst preserving long-term cell viability. In subsequent work, the researchers studied more in detail the effect of the addition of PEI during the fabrication process of HA-alginate bioinks for 3D printing of Schwann cells and chondrocytes. ${ }^{150}$ This polycation has a stabilising effect on the $3 \mathrm{D}$ constructs due to the formation of a polyelectrolyte complex with the alginate chains. Moreover, it binds ionically to HA forming a complex between its amino groups and the carboxylic groups of HA. The bioink formulation was prepared by combining alginate $(2.5 \% \mathrm{wt} / \mathrm{vol})$ with hyaluronic acid $(0.25 \%$ wt/vol) in 0.3 M sucrose and HEPES (25 mM). PVA was also added to the cross-linking solution $\left(100 \mathrm{mM} \mathrm{CaCl}_{2}\right)$ to further increase the viscosity and prevent the printed constructs to float in the cross-linking solution. The performed experiments demonstrated that the treatment with PEI $(0.1 \%, 0.2 \%$ or $0.5 \% \mathrm{wt} / \mathrm{vol})$ improved the mechanical properties of the bioink formulation and reduced the scaffold degradation rate and degree of swelling. However, the survival rate of the cells encapsulated in the bioink tended to decrease with increasing PEI concentrations. It is well-known that polyamines are relatively toxic to cells. ${ }^{151}$ Therefore, optimal concentrations of the polycation are required to guarantee optimal outcomes and applicability of the bioinks. This study clearly demonstrates how highly complex formulations can emerge in this field of research. This is obviously an advantage of this approach to tissue engineering, but it is vital to carefully characterise the impact of each component in the system in order to best optimise the overall gel for the desired application.

More recently, these researchers developed a more complex bioink formulation composed of hyaluronic acid, RGD-modified alginate and fibrin for 3D bioprinting of Schwann cell scaffolds. ${ }^{76}$ This bioink was prepared by binding alginate to RGD peptide in a 250:1 ratio by coupling reaction in the presence of ethyl(dimethylaminopropyl) carbodiimide (EDC) and $\mathrm{N}$-hydroxysuccinimide (NHS) and subsequently combining it with hyaluronic acid to obtain a final $2.0 \% \mathrm{wt} / \mathrm{vol}$ concentration of alginate and $1.0 \%$ wt/vol modified-HA. To evaluate the effect of cross-linker concentration on the stability and shape fidelity of the 3D printed constructs, they used different $\mathrm{CaCl}_{2}$ concentrations $(10,20,30$ and $40 \mathrm{mM}$ ). The collected results showed that the cross-linker concentration and bioprinting speed had a remarkable effect on the shape, stability and pore size of the printed structures. Increasing the $\mathrm{Ca}^{2+}$ concentrations resulted in better shape fidelity, however excessively high concentrations could result in structural failure. This is due to the formation of a very rigid structure in a very short time, which reduces the attachment of subsequently printed hydrogel layers and hence the stability of the 3D printed construct. The obtained hydrogels could support Schwann cell viability and function and were shown to be promising for nerve regeneration.

Cartilage scaffolds. The applicability of HA-alginate based bioinks for the fabrication of articular cartilaginous constructs was explored by Antich et al. ${ }^{152}$ They prepared a bioink formulation containing $1.0 \% \mathrm{wt} / \mathrm{vol} \mathrm{HA}$ and $2.0 \% \mathrm{wt} / \mathrm{vol}$ alginate, which was cross-linked with a $100 \mathrm{mM} \mathrm{CaCl}_{2}$ solution. The polymer blend displayed good printability, gelling ability, optimal stiffness and degradability. To further improve the stability of the 3D printed constructs and ensure optimal mechanical properties for the growth of chondrocytes, the bioink was deposited on a biodegradable polylactic acid (PLA) framework and subsequently cross-linked. The resulting scaffold showed biomimetic mechanical properties and promoted chondrogenesis - it is therefore a promising material for articular cartilage regeneration.

\section{Design of alginate-based bioink formulations for specific applications}

3D bioprinting allows the fabrication of complex tissue constructs, including 3D scaffolds, hollow tubes and organs. This technology is rapidly evolving towards functional tissues and implants for tissue regeneration in vivo applications. ${ }^{153-155}$ The bioink formulations we have discussed in this review have been applied for the fabrication of different 3D scaffolds (e.g. cartilage, bone, fibroblasts, endothelial and neural), indeed some of these applications have been noted earlier in the review. The majority of the studies we have reported here have been focused on the bioink properties, printability and compatibility with specific cell lines. These are crucial elements to consider to achieve more complex $3 \mathrm{D}$ structures for in vivo applications. $^{153}$

In this section, we will try to use this information to generate a set of design rules to help the design of alginate-based bioinks for specific cellular types (Fig. 7). We described in detail the composition of different multi-component alginate/ polysaccharide formulations used for the development of different 3D scaffolds; therefore, all the specifics of the different alginate/polysaccharide blends (e.g. concentrations of the different components, type and concentration of cross-linker) will not be discussed again, but we will instead provide a high-level overview of the available formulations for specific applications. The reader is referred to the previous sections to find more detailed descriptions of the bioink composition.

Different elements should be taken into consideration when designing a bioink for a specific application (Fig. 7). In general these should be considered in the following order to develop the optimal formulation:

1. What type of alginate should be used? What is the optimal concentration? What type of cross-linker is ideal and at which concentration?

2. Does the addition of another polysaccharide improve the mechanical properties, stability and degradation profile of the bioink formulation?

3. Does the incorporation of other additives (e.g. growth factors or nanoparticles) improve biocompatibility or aid cell differentiation? 


\begin{tabular}{|c|c|c|c|}
\hline BIOINK DESIGN IN THREE STEPS & \multicolumn{3}{|c|}{ WHICH ADDITIONAL POLYSACCHARIDE? } \\
\hline \multirow{4}{*}{$\begin{array}{l}\text { 1. CHOICE OF ALGINATE AND CROSS-LINKER: } \\
\text { - Choose molecular weight, viscosity and } \\
\text { alginate concentration } \\
\text { - Choose type and concentration of cross-linker } \\
\text { 2. CHOICE OF ADDITIONAL POLYSACCHARIDE } \\
\text { Choose an additional polysaccharide that can } \\
\text { improve the mechanical properties, stability and } \\
\text { degradation profile of the bioink formulation }\end{array}$} & Alginate & Alginate/Agarose & Alginate/Cellulose \\
\hline & $\begin{array}{l}\text { - } \text { Adipose tissue } \\
\text { - } \text { Bone tissue } \\
\text { - } \text { Chondrocytes/Cartilage } \\
\text { - } \text { Fibroblasts/vascular } \\
\text { constructs } \\
\text { - Hepatocytes } \\
\text { - } \text { Mesenchymal stem cells } \\
\text { - Neural tissue }\end{array}$ & $\begin{array}{l}\text { - } \text { Chondrocytes/Cartilage } \\
\text { - } \text { Endothelial cells } \\
\text { - } \text { Mesenchymal stem cells } \\
\text { tissue }\end{array}$ & $\begin{array}{l}\text { - Adipose tissue derived } \\
\text { stem cells } \\
\text { - Chondrocytes/Cartilage } \\
\text { - Mesenchymal stem cells } \\
\text { - Pluripotent stem cells }\end{array}$ \\
\hline & Alginate/Methylcellulose & Alginate/Gellan gum & Alginate/Hyaluronic acid \\
\hline & $\begin{array}{l}\text { - Chondrocytes/Cartilage } \\
\text { Fibroblasts } \\
\text { - Mesenchymal stem cells } \\
\text { - Pancreatic cells } \\
\text { - Plant cells }\end{array}$ & $\begin{array}{ll}\text { - } & \text { Bone tissue } \\
\text { - } & \text { Chondrocytes/Cartilage } \\
\text { - } & \text { Mesenchymal stem cells }\end{array}$ & $\begin{array}{l}\text { - Chondrocytes/Cartilage } \\
\text { - Neural tissue/Schwann } \\
\text { cells }\end{array}$ \\
\hline
\end{tabular}

Fig. 7 Design of alginate/polysaccharide bioinks and main applications of the different formulations.

It is well known that hydrogel scaffolds should mimic the mechanical properties of native tissues to provide an optimal environment to the cells. ${ }^{22,156}$ Therefore soft tissues, such as neural tissue, preferentially grow on soft hydrogels. ${ }^{38}$ We have described various examples of bioink formulations for neural scaffolds in which alginate was the only component. ${ }^{35,73-78}$ Such scaffolds were fabricated using alginate concentrations between $0.5-3.0 \% \mathrm{wt} / \mathrm{vol}$, with the best results in terms of cell growth at low alginate concentrations. ${ }^{75,76}$ The use of gelatin sacrificial templates allowed materials with higher mechanical stability and better shape fidelity. ${ }^{77,78}$ The combination of alginate with other polysaccharides is a way of avoiding the use of sacrificial templates. Examples of multicomponent bioinks were obtained by combining alginate with agarose $(1.5 \% \mathrm{wt} / \mathrm{vol})^{87,88}$ or hyaluronic acid $(0.25 \% \mathrm{wt} / \mathrm{vol}),{ }^{76,149,150}$ which provided the necessary mechanical support to the 3D printed structures (Fig. 7).

Fibroblasts, vascular and endothelial constructs can be fabricated using alginate bioinks prepared with $2.0-6.0 \% \mathrm{wt} / \mathrm{vol}$ alginate concentrations. ${ }^{61,71,72,157}$ Again, combination with other polysaccharides such as agarose $(0.6-2.4 \% \mathrm{wt} / \mathrm{vol})^{89}$ or methylcellulose $(9.0 \% \mathrm{wt} / \mathrm{vol})^{114}$ is an option to obtain bioinks with good extrudability, thixotropic behaviour and enhanced stability (Fig. 7).

Alginate bioinks can also be used for the 3D printing of mesenchymal stem cells that can then differentiate into different lines forming (for example) cartilage $^{70}$ or bone scaffolds. ${ }^{65,66,69}$ In most of the reported examples, these hard tissue scaffolds were obtained using an alginate concentration between $2.0-3.0 \% \mathrm{wt} / \mathrm{vol}$ in combination with other additives such as graphene oxide, hydroxyapatite or growth factors. The preparation of polysaccharide blends using agarose, cellulose, methylcellulose, gellan gum and hyaluronic acid can also be a successful strategy to improve the mechanical properties of the resulting 3D printed structures and their stability and integrity over time (Fig. 7). Bioinks based on alginate blends with cellulose $(2.0 \% \mathrm{wt} / \mathrm{vol})^{102,105,108,109}$ and methylcellulose $(9.0 \% \mathrm{wt} / \mathrm{vol})^{115-118}$ are the most studied and gave promising results without the need for functional additives. Other polysaccharides such as agarose, ${ }^{81,85}$ gellan gum ${ }^{126,127,132,138}$ and hyaluronic acid ${ }^{152}$ can also be combined with alginate, but the use of sacrificial templates or other components (e.g. growth factors, silica nanoparticles or calcium phosphate cement) may be required to improve the bioink performance.

Finally, 3D printing of other types of tissues (e.g. hepatic or pancreatic) has also been achieved using alginate (Fig. 7). ${ }^{79,119}$ However, relatively few research studies have been performed in this area and, therefore, this may be of interest for further investigation.

When designing a bioink formulation, it is important to keep in mind that functional additives can be added to improve biocompatibility, performance and suitability of the bioink for a specific application. We have highlighted various examples throughout the text, which included the use of growth factors (e.g. TGF- $\beta$ or VEGF), graphene oxide, hydroxyapatite, LAPONITE $^{\mathbb{R}}$, silica nanoparticles, etc. $^{66,70,117,118,138}$ The encapsulation of functional additives into alginate bioinks could also, therefore, be considered. This topic falls outside the scope of this review, however relevant readings can be found elsewhere. ${ }^{19,158-164}$

\section{Conclusions}

3D bioprinting of cellular constructs is a rapidly expanding research area. Alginate is a natural, biocompatible polymer that has been widely applied in 3D bioprinting. Most of the reported bioink formulations based on pure alginate were employed to $3 \mathrm{D}$ print stem cells, fibroblasts, neurons and hepatocytes. Alginate has good biocompatibility, and the performance can be tailored by tuning the molecular weight of the polymer as well as its loading, the concentration of the divalent metal crosslinking agent, and the type of crosslinking agent used. In particular, sources of calcium ions with low solubility have been used to slowly crosslink alginate systems and generate more homogeneous materials. 
The properties of alginate-based bioink formulations can also be tailored by combination with other polysaccharides, such as agarose, cellulose, methylcellulose, gellan gum and hyaluronic acid. The preparation of polymer blends allows, in particular, the mechanical properties and stability of the resulting $3 \mathrm{D}$ printed constructs to be improved, expanding the range of applications of alginate bioinks in tissue engineering and regenerative medicine. For different biological applications, it is vital to tailor the materials properties of the printed object for the best outcomes, and a multi-component approach using alginate gels is a powerful way of achieving this. In some cases, alginate can be combined with sacrificial materials, which can temporarily reinforce the $3 \mathrm{D}$ printed object, while subsequently being removed either through processes such as melting or biodegradation. This can provide a degree of temporal control over the $3 \mathrm{D}$ printed materials. We suggest that such control will become increasingly important in next generation tissue engineering, where longer-term fates of cellular tissue will need to be directed.

In addition to blending with different polysaccharides, in a number of cases, other functional additives are also incorporated into 3D-printed alginate systems. For example, nanoparticles and clays can have significant impacts on mechanical performance, biocompatible peptides, such as RGD can improve cellular proliferation, and by incorporating growth factors, more subtle effects on cellular proliferation and differentiation can be exerted. We suggest that the ease of formulating multiple components into these gels offers them considerable potential for further development.

In summary, alginate-based bioinks are promising materials, and we hope that this review will facilitate the identification of different alginate-polysaccharide bioink formulations, their optimal applications and will thus help to inform the design of second generation bioinks, allowing this relatively simple gel system to achieve more sophisticated control over biological processes.

\section{Conflicts of interest}

The authors declare no conflicts of interest.

\section{Acknowledgements}

We thank EPSRC for funding (EP/P03361X/1).

\section{References}

1 M. Mobaraki, M. Ghaffari, A. Yazdanpanah, Y. Luo and D. K. Mills, Bioprinting, 2020, 18, e00080.

2 E. Saygili, A. A. Dogan-Gurbuz, O. Yesil-Celiktas and M. S. Draz, Bioprinting, 2020, 18, e00071.

3 J. A. Semba, A. A. Mieloch and J. D. Rybka, Bioprinting, 2020, 18, e00070.

4 T. Bedir, S. Ulag, C. B. Ustundag and O. Gunduz, Mater. Sci. Eng., C, 2020, 110, 110741.

5 J. M. Unagolla and A. C. Jayasuriya, Appl. Mater. Today, 2020, 18, 100479.
6 I. Matai, G. Kaur, A. Seyedsalehi, A. McClinton and C. T. Laurencin, Biomaterials, 2020, 226, 119536.

7 Y. Huang, X.-F. Zhang, G. Gao, T. Yonezawa and X. Cui, Biotechnol. J., 2017, 12, 1600734.

8 E. Axpe and M. L. Oyen, Int. J. Mol. Sci., 2016, 17, 1976.

9 P. Rastogi and B. Kandasubramanian, Biofabrication, 2019, 11, 042001.

10 A. C. Hernández-González, L. Téllez-Jurado and L. M. Rodríguez-Lorenzo, Carbohydr. Polym., 2020, 229, 115514.

11 J. Jang, J. Y. Park, G. Gao and D. W. Cho, Biomaterials, 2018, 156, 88-106.

12 S. Das and B. Basu, J. Ind. Inst. Sci., 2019, 99, 405-428.

13 P. S. Gungor-Ozkerim, I. Inci, Y. S. Zhang, A. Khademhosseini and M. R. Dokmeci, Biomater. Sci., 2018, 6, 915-946.

14 X. Du, J. Zhou, J. Shi and B. Xu, Chem. Rev., 2015, 115, 13165-13307.

15 W. Aljohani, M. W. Ullah, X. Zhang and G. Yang, Int. J. Biol. Macromol., 2018, 107, 261-275.

16 S. J. Bidarra, C. C. Barrias and P. L. Granja, Acta Biomater., 2014, 10, 1646-1662.

17 G. Kaklamani, D. Cheneler, L. M. Grover, M. J. Adams and J. Bowen, J. Mech. Behav. Biomed., 2014, 36, 135-142.

18 H. Martínez Ávila, S. Schwarz, N. Rotter and P. Gatenholm, Bioprinting, 2016, 1-2, 22-35.

19 J. Jia, D. J. Richards, S. Pollard, Y. Tan, J. Rodriguez, R. P. Visconti, T. C. Trusk, M. J. Yost, H. Yao, R. R. Markwald and Y. Mei, Acta Biomater., 2014, 10, 4323-4331.

$20 \mathrm{H}$. Onoe, T. Okitsu, A. Itou, M. Kato-Negishi, R. Gojo, D. Kiriya, K. Sato, S. Miura, S. Iwanaga, K. KuribayashiShigetomi, Y. T. Matsunaga, Y. Shimoyama and S. Takeuchi, Nat. Mater., 2013, 12, 584-590.

21 K. Akeda, A. Nishimura, H. Satonaka, K. Shintani, K. Kusuzaki, A. Matsumine, Y. Kasai, K. Masuda and A. Uchida, Oncol. Rep., 2009, 22, 997-1003.

22 T. Andersen, P. Auk-Emblem and M. Dornish, Microarrays, 2015, 4, 133-161.

23 X. B. Huang, X. Y. Zhang, X. G. Wang, C. Wang and B. Tang, J. Biosci. Bioeng., 2012, 114, 1-8.

24 C. Tai, S. Bouissil, E. Gantumur, M. S. Carranza, A. Yoshii, S. Sakai, G. Pierre, P. Michaud and C. Delattre, Appl. Sci., 2019, 9, 2596.

25 R. Suntornnond, J. An and C. K. Chua, Macromol. Mater. Eng., 2017, 302, 1600266.

26 E. Davoodi, E. Sarikhani, H. Montazerian, S. Ahadian, M. Costantini, W. Swieszkowski, S. M. Willerth, K. Walus, M. Mofidfar, E. Toyserkani, A. Khademhosseini and N. Ashammakhi, Adv. Mater. Technol., 2020, 1901044.

27 J. Y. Ma, Y. C. Wang and J. Liu, RSC Adv., 2018, 8, 21712-21727.

28 J. P. Li, M. J. Chen, X. Q. Fan and H. F. Zhou, J. Transl. Med., 2016, 14, 271.

29 T. Jiang, J. G. Munguia-Lopez, S. Flores-Torres, J. Kort-Mascort and J. M. Kinsella, Appl. Phys. Rev., 2019, 6, 011310.

30 E. S. Bishop, S. Mostafa, M. Pakvasa, H. H. Luu, M. J. Lee, J. M. Wolf, G. A. Ameer, T. C. He and R. R. Reid, Genes Diseases, 2017, 4, 185-195. 
31 S. J. Song, J. Choi, Y. D. Park, S. Hong, J. J. Lee, C. B. Ahn, H. Choi and K. Sun, Artif. Organs, 2011, 35, 1132-1136.

32 L. Raddatz, A. Lavrentieva, I. Pepelanova, J. Bahnemann, D. Geier, T. Becker, T. Scheper and S. Beutel, J. Funct. Biomater., 2018, 9, 4.

33 R. T. Xiong, Z. Y. Zhang and Y. Huang, J. Manuf. Process., 2015, 20, 450-455.

34 J. Y. Yan, Y. Huang and D. B. Chrisey, Biofabrication, 2013, 5, 015002.

35 M. Matyash, F. Despang, C. Ikonomidou and M. Gelinsky, Tissue Eng., Part C, 2013, 20, 401-411.

36 A. D. Augst, H. J. Kong and D. J. Mooney, Macromol. Biosci., 2006, 6, 623-633.

37 H. J. Kong, M. K. Smith and D. J. Mooney, Biomaterials, 2003, 24, 4023-4029.

38 A. Banerjee, M. Arha, S. Choudhary, R. S. Ashton, S. R. Bhatia, D. V. Schaffer and R. S. Kane, Biomaterials, 2009, 30, 4695-4699.

39 J. Park, S. J. Lee, S. Chung, J. H. Lee, W. D. Kim, J. Y. Lee and S. A. Park, Mater. Sci. Eng., C, 2017, 71, 678-684.

40 K. Y. Lee and D. J. Mooney, Prog. Polym. Sci., 2012, 37, 106-126.

41 W. X. Jiao, W. X. Chen, Y. Q. Mei, Y. H. Yun, B. Q. Wang, Q. P. Zhong, H. M. Chen and W. J. Chen, Molecules, 2019, 24, 4374.

42 F. E. Freeman and D. J. Kelly, Sci. Rep., 2017, 7, 17042.

43 L. D. Loozen, F. Wegman, F. C. Öner, W. J. A. Dhert and J. Alblas, J. Mater. Chem. B, 2013, 1, 6619-6626.

44 J. Zhang, E. Wehrle, J. R. Vetsch, G. R. Paul, M. Rubert and R. Müller, Biomed. Mater., 2019, 14, 065009.

45 H. Xu, Z. Zhang and C. Xu, J. Appl. Phys., 2019, 125, 114901. 46 C. K. Kuo and P. X. Ma, Biomaterials, 2001, 22, 511-521.

47 S. Naghieh, M. R. Karamooz-Ravari, M. D. Sarker, E. Karki and X. Chen, J. Mech. Behav. Biomed., 2018, 80, 111-118.

48 A. G. Tabriz, M. A. Hermida, N. R. Leslie and W. Shu, Biofabrication, 2015, 7, 045012.

49 T. Y. Wong, L. A. Preston and N. L. Schiller, Ann. Rev. Microbiol., 2000, 54, 289-340.

50 T. Boontheekul, H. J. Kong and D. J. Mooney, Biomaterials, 2005, 26, 2455-2465.

51 L. Y. Chen, R. Z. Shen, S. Komasa, Y. X. Xue, B. Y. Jin, Y. P. Hou, J. Okazaki and J. Gao, Int. J. Mol. Sci., 2017, 18, 989.

52 H. J. Kong, D. Kaigler, K. Kim and D. J. Mooney, Biomacromolecules, 2004, 5, 1720-1727.

53 T. V. Shkand, M. O. Chizh, I. V. Sleta, B. P. Sandomirsky, A. L. Tatarets and L. D. Patsenker, Methods Appl. Fluores., 2016, 4, 4.

54 J. S. Yang, Y. J. Xie and W. He, Carbohydr. Polym., 2011, 84, 33-39.

55 S. Reakasame and A. R. Boccaccini, Biomacromolecules, 2018, 19, 3-21.

56 M. Y. Teo, S. Kee, N. RaviChandran, L. Stuart, K. C. Aw and J. Stringer, ACS Appl. Mater. Interfaces, 2020, 12, 1832-1839.

57 J. Dohnal and F. Stepanek, Powder Technol., 2010, 200, 254-259.
58 J. H. Y. Chung, S. Naficy, G. G. Wallace and S. O'Leary, Adv. Polym. Technol., 2016, 35, 439-446.

59 J. T. Delaney, A. R. Liberski, J. Perelaer and U. S. Schubert, Soft Matter, 2010, 6, 866-869.

60 Y. S. Zhang, Q. M. Pi and A. M. van Genderen, JoVE J. Vis. Exp., 2017, 126, e55957.

61 Q. Gao, Z. Liu, Z. Lin, J. Qiu, Y. Liu, A. Liu, Y. Wang, M. Xiang, B. Chen, J. Fu and Y. He, ACS Biomater. Sci. Eng., 2017, 3, 399-408.

62 T. R. Cuadros, O. Skurtys and J. M. Aguilera, Carbohydr. Polym., 2012, 89, 1198-1206.

63 S. Ghorbanian, M. A. Qasaimeh, M. Akbari, A. Tamayol and D. Juncker, Biomed. Microdevices, 2014, 16, 387-395.

64 H. M. Zhao, Y. S. Chen, L. Shao, M. J. Xie, J. Nie, J. J. Qiu, P. Zhao, H. Ramezani, J. Z. Fu, H. W. Ouyang and Y. He, Small, 2018, 14, 1802630.

65 G. Choe, S. Oh, J. M. Seok, S. A. Park and J. Y. Lee, Nanoscale, 2019, 11, 23275-23285.

66 T. T. Demirtaş, G. Irmak and M. Gümüşderelioğlu, Biofabrication, 2017, 9, 035003.

67 S. Mondal and U. Pal, J. Drug Delivery Sci. Technol., 2019, 53, 101131.

68 G. Wei, C. Gong, K. Hu, Y. Wang and Y. Zhang, Nanomaterials, 2019, 9, 1435.

69 B. E. J. Lee, A. Shahin-Shamsabadi, M. K. Wong, S. Raha, P. R. Selvaganapathy and K. Grandfield, Adv. Biosyst., 2019, 3, 1900126.

70 S. Rathan, L. Dejob, R. Schipani, B. Haffner, M. E. Möbius and D. J. Kelly, Adv. Healthcare Mater., 2019, 8, 1801501.

71 R. Xiong, Z. Zhang, W. Chai, Y. Huang and D. B. Chrisey, Biofabrication, 2015, 7, 045011.

72 E. Y. S. Tan and W. Y. Yeong, Int. J. Bioprint., 2015, 1, 49-56.

73 S. Grijalvo, M. Nieto-Díaz, R. M. Maza, R. Eritja and D. D. Díaz, Biotechnol. J., 2019, 14, 1900275.

74 M. Matyash, F. Despang, R. Mandal, D. Fiore, M. Gelinsky and C. Ikonomidou, Tissue Eng., Part A, 2011, 18, 55-66.

75 L. Ning, Y. Xu, X. Chen and D. J. Schreyer, J. Biomater. Sci., Polym. Ed., 2016, 27, 898-915.

76 L. Ning, N. Zhu, F. Mohabatpour, M. D. Sarker, D. J. Schreyer and X. Chen, J. Mater. Chem. B, 2019, 7, 4538-4551.

77 S. Naghieh, M. D. Sarker, E. Abelseth and X. Chen, J. Mech. Behav. Biomed., 2019, 93, 183-193.

78 J. Lewicki, J. Bergman, C. Kerins and O. Hermanson, Bioprinting, 2019, 16, e00053.

79 A. Faulkner-Jones, C. Fyfe, D. J. Cornelissen, J. Gardner, J. King, A. Courtney and W. Shu, Biofabrication, 2015, 7, 044102.

80 S. Graham, P. F. Marina and A. Blencowe, Carbohydr. Polym., 2019, 207, 143-159.

81 G. R. López-Marcial, A. Y. Zeng, C. Osuna, J. Dennis, J. M. García and G. D. O'Connell, ACS Biomater. Sci. Eng., 2018, 4, 3610-3616.

82 E. Mirdamadi, N. Muselimyan, P. Koti, H. Asfour and N. Sarvazyan, 3D Print. Addit. Manuf., 2019, 6, 158-164. 
83 P. Zarrintaj, S. Manouchehri, Z. Ahmadi, M. R. Saeb, A. M. Urbanska, D. L. Kaplan and M. Mozafari, Carbohydr. Polym., 2018, 187, 66-84.

84 H. F. Darge, A. T. Andrgie, H. C. Tsai and J. Y. Lai, Int. J. Biol. Macromol., 2019, 133, 545-563.

85 A. C. Daly, S. E. Critchley, E. M. Rencsok and D. J. Kelly, Biofabrication, 2016, 8, 045002.

86 G. R. López-Marcial, A. Y. Zeng, C. Osuna, J. Dennis, J. M. García and G. D. O'Connell, ACS Biomater. Sci. Eng., 2018, 4, 3610-3616.

87 Q. Gu, E. Tomaskovic-Crook, R. Lozano, Y. Chen, R. M. Kapsa, Q. Zhou, G. G. Wallace and J. M. Crook, Adv. Healthcare Mater., 2016, 5, 1429-1438.

88 Q. Gu, E. Tomaskovic-Crook, G. G. Wallace and J. M. Crook, Adv. Healthcare Mater., 2017, 6, 1700175.

89 Q. Zou, B. E. Grottkau, Z. He, L. Shu, L. Yang, M. Ma and C. Ye, Mater. Sci. Eng., C, 2020, 108, 110205.

90 D. Klemm, F. Kramer, S. Moritz, T. Lindström, M. Ankerfors, D. Gray and A. Dorris, Angew. Chem. Int. Ed., 2011, 50, 5438-5466.

91 H. Luo, R. Cha, J. Li, W. Hao, Y. Zhang and F. Zhou, Carbohydr. Polym., 2019, 224, 115144.

92 S. Naz, J. S. Ali and M. Zia, Bio-Des. Manufact., 2019, 2, 187-212.

93 L. H. Nguyen, S. Naficy, R. Chandrawati and F. Dehghani, Adv. Mater. Interfaces, 2019, 6, 1900424.

94 P. Phanthong, P. Reubroycharoen, X. Hao, G. Xu, A. Abudula and G. Guan, Carbon Resour. Convers., 2018, 1, 32-43.

95 S. Salimi, R. Sotudeh-Gharebagh, R. Zarghami, S. Y. Chan and K. H. Yuen, ACS Sustainable Chem. Eng., 2019, 7, 15800-15827.

96 H. Voisin, L. Bergström, P. Liu and A. P. Mathew, Nanomaterials, 2017, 7, 57.

97 L. Huang, X. Du, S. Fan, G. Yang, H. Shao, D. Li, C. Cao, Y. Zhu, M. Zhu and Y. Zhang, Carbohydr. Polym., 2019, 221, 146-156.

98 W. Xu, B. Z. Molino, F. Cheng, P. J. Molino, Z. Yue, D. Su, X. Wang, S. Willför, C. Xu and G. G. Wallace, ACS Appl. Mater. Interfaces, 2019, 11, 8838-8848.

99 M. Ojansivu, A. Rashad, A. Ahlinder, J. Massera, A. Mishra, K. Syverud, A. Finne-Wistrand, S. Miettinen and K. Mustafa, Biofabrication, 2019, 11, 035010.

100 S. Sultan, G. Siqueira, T. Zimmermann and A. P. Mathew, Curr. Opin. Biomed. Eng., 2017, 2, 29-34.

101 C. C. Piras, S. Fernández-Prieto and W. M. De Borggraeve, Biomater. Sci., 2017, 5, 1988-1992.

102 K. Markstedt, A. Mantas, I. Tournier, H. Martínez Ávila, D. Hägg and P. Gatenholm, Biomacromolecules, 2015, 16, 1489-1496.

103 M. Müller, E. Öztürk, Ø. Arlov, P. Gatenholm and M. Zenobi-Wong, Ann. Biomed. Eng., 2017, 45, 210-223.

104 K. Säljö, L. S. Orrhult, P. Apelgren, K. Markstedt, L. Kölby and P. Gatenholm, Bioprinting, 2020, 17, e00065.

105 D. Nguyen, D. A. Hägg, A. Forsman, J. Ekholm, P. Nimkingratana, C. Brantsing, T. Kalogeropoulos, S. Zaunz,
S. Concaro, M. Brittberg, A. Lindahl, P. Gatenholm, A. Enejder and S. Simonsson, Sci. Rep., 2017, 7, 658.

106 R. C. Gupta, R. Lall, A. Srivastava and A. Sinha, Front. Vet. Sci., 2019, 6, 192.

107 N. S. Hwang, S. Varghese, Z. Zhang and J. Elisseeff, Tissue Eng., 2006, 12, 2695-2706.

108 P. Apelgren, M. Amoroso, A. Lindahl, C. Brantsing, N. Rotter, P. Gatenholm and L. Kölby, PLoS One, 2017, 12, e0189428.

109 T. Möller, M. Amoroso, D. Hägg, C. Brantsing, N. Rotter, P. Apelgren, A. Lindahl, L. Kölby and P. Gatenholm, Plast. Reconstruct. Surg., 2017, 5, e1227.

110 S. Morozova, Polym. Int., 2020, 69, 125-130.

111 P. L. Nasatto, F. Pignon, J. L. M. Silveira, M. E. R. Duarte, M. D. Noseda and M. Rinaudo, Polymers, 2015, 7, 777-803.

112 A. Forghani and R. Devireddy, Methods Mol. Biol., 2018, 1773, 41-51.

113 S. Naghieh, M. D. Sarker, N. K. Sharma, Z. Barhoumi and X. Chen, Appl. Sci., 2020, 10, 292.

114 H. Li, Y. J. Tan, K. F. Leong and L. Li, ACS Appl. Mater. Interfaces, 2017, 9, 20086-20097.

115 K. Schütz, A.-M. Placht, B. Paul, S. Brüggemeier, M. Gelinsky and A. Lode, J. Tissue Eng. Regener. Med., 2017, 11, 1574-1587.

116 E. Hodder, S. Duin, D. Kilian, T. Ahlfeld, J. Seidel, C. Nachtigall, P. Bush, D. Covill, M. Gelinsky and A. Lode, J. Mater. Sci.: Mater. Med., 2019, 30, 10.

117 T. Ahlfeld, G. Cidonio, D. Kilian, S. Duin, A. R. Akkineni, J. I. Dawson, S. Yang, A. Lode, R. O. C. Oreffo and M. Gelinsky, Biofabrication, 2017, 9, 034103.

118 T. Ahlfeld, F. Doberenz, D. Kilian, C. Vater, P. Korn, G. Lauer, A. Lode and M. Gelinsky, Biofabrication, 2018, 10, 045002.

119 S. Duin, K. Schütz, T. Ahlfeld, S. Lehmann, A. Lode, B. Ludwig and M. Gelinsky, Adv. Healthcare Mater., 2019, 8, 1801631.

120 A. Lode, F. Krujatz, S. Brüggemeier, M. Quade, K. Schütz, S. Knaack, J. Weber, T. Bley and M. Gelinsky, Eng. Life Sci., 2015, 15, 177-183.

121 M. Das and T. K. Giri, J. Drug Delivery Sci. Technol., 2020, 56, 101586.

122 K. M. Zia, S. Tabasum, M. F. Khan, N. Akram, N. Akhter, A. Noreen and M. Zuber, Int. J. Biol. Macromol., 2018, 109, 1068-1087.

123 A. M. Fialho, L. M. Moreira, A. T. Granja, A. O. Popescu, K. Hoffmann and I. Sá-Correia, Appl. Microbiol. Biotechnol., 2008, 79, 889.

124 R. M. Banik, B. Kanari and S. N. Upadhyay, World J. Microbiol. Biotechnol., 2000, 16, 407-414.

125 F. S. Palumbo, S. Federico, G. Pitarresi, C. Fiorica and G. Giammona, Carbohydr. Polym., 2020, 229, 115430.

126 M. Kesti, C. Eberhardt, G. Pagliccia, D. Kenkel, D. Grande, A. Boss and M. Zenobi-Wong, Adv. Funct. Mater., 2015, 25, 7406-7417.

127 M. Lee, K. Bae, P. Guillon, J. Chang, O. Arlov and M. Zenobi-Wong, ACS Appl. Mater. Interfaces, 2018, 10, 37820-37828. 
128 Z. Azimi Dijvejin, A. Ghaffarkhah, S. Sadeghnejad and M. Vafaie Sefti, Polym. J., 2019, 51, 693-707.

129 C. Dannert, B. T. Stokke and R. S. Dias, Polymers, 2019, 11, 275.

130 P. Thoniyot, M. J. Tan, A. A. Karim, D. J. Young and X. J. Loh, Adv. Sci., 2015, 2, 1400010.

131 A. Boonmahitthisud, L. Nakajima, K. D. Nguyen and T. Kobayashi, J. Appl. Polym. Sci., 2017, 134, 44557.

132 A. R. Akkineni, T. Ahlfeld, A. Funk, A. Waske, A. Lode and M. Gelinsky, Polymers, 2016, 8, 170.

133 H. Widerøe and S. Danielsen, Naturwissenschaften, 2001, 88, 224-228.

134 Ý. A. Mørch, I. Donati and B. L. Strand, Biomacromolecules, 2006, 7, 1471-1480.

135 F. Yang, C. G. Williams, D.-A. Wang, H. Lee, P. N. Manson and J. Elisseeff, Biomaterials, 2005, 26, 5991-5998.

136 E. Mauri, A. Sacchetti, N. Vicario, L. Peruzzotti-Jametti, F. Rossi and S. Pluchino, Biomater. Sci., 2018, 6, 501-510.

137 J. Dumbleton, P. Agarwal, H. Huang, N. Hogrebe, R. Han, K. J. Gooch and X. He, Cell. Mol. Bioeng., 2016, 9, 277-288.

138 T. Ahlfeld, F. P. Schuster, Y. Förster, M. Quade, A. R. Akkineni, C. Rentsch, S. Rammelt, M. Gelinsky and A. Lode, Adv. Healthcare Mater., 2019, 8, 1801512.

139 X. Xu, A. K. Jha, D. A. Harrington, M. C. Farach-Carson and X. Jia, Soft Matter, 2012, 8, 3280-3294.

140 E. Ahmadian, A. Eftekhari, S. M. Dizaj, S. Sharifi, M. Mokhtarpour, A. N. Nasibova, R. Khalilov and M. Samiei, Int. J. Biol. Macromol., 2019, 140, 245-254.

141 E. Ahmadian, S. M. Dizaj, A. Eftekhari, E. Dalir, P. Vahedi, A. Hasanzadeh and M. Samiei, Drug Res., 2020, 70, 6-11.

142 E. Hachet, H. Van Den Berghe, E. Bayma, M. R. Block and R. Auzély-Velty, Biomacromolecules, 2012, 13, 1818-1827.

143 C. B. Highley, G. D. Prestwich and J. A. Burdick, Curr. Opin. Biotechnol, 2016, 40, 35-40.

144 H. Li, Z. Qi, S. Zheng, Y. Chang, W. Kong, C. Fu, Z. Yu, X. Yang and S. Pan, Adv. Mater. Sci. Eng., 2019, 2019, 3027303.

145 C. C. Clark, J. Aleman, L. Mutkus and A. Skardal, Bioprinting, 2019, 16, e00058.

146 E. A. Kiyotake, A. W. Douglas, E. E. Thomas, S. L. Nimmo and M. S. Detamore, Acta Biomater., 2019, 95, 176-187.
147 T. Lam, T. Dehne, J. P. Krüger, S. Hondke, M. Endres, A. Thomas, R. Lauster, M. Sittinger and L. Kloke, J. Biomed. Mater. Res. B, 2019, 107, 2649-2657.

148 Y. Qiao, S. Xu, T. Zhu, N. Tang, X. Bai and C. Zheng, Polymer, 2020, 186, 121994.

149 A. Rajaram, D. Schreyer and D. Chen, 3D Print. Addit. Manuf., 2014, 1, 194-203.

150 A. Rajaram, D. J. Schreyer and D. X. B. Chen, J. Biomater. Sci., Polym. Ed., 2015, 26, 433-445.

151 A. E. Pegg, Chem. Res. Toxicol., 2013, 26, 1782-1800.

152 C. Antich, J. de Vicente, G. Jiménez, C. Chocarro, E. Carrillo, E. Montañez, P. Gálvez-Martín and J. A. Marchal, Acta Biomater., 2020, 106, 114-123.

153 S. V. Murphy and A. Atala, Nat. Biotechnol., 2014, 32, 773-785.

154 F. Pati, J. Gantelius and H. A. Svahn, Angew. Chem. Int. Ed., 2016, 55, 4650-4665.

155 S. Vijayavenkataraman, W. C. Yan, W. F. Lu, C. H. Wang and J. Y. H. Fuh, Adv. Drug Delivery Rev., 2018, 132, 296-332.

156 A. Seidi, M. Ramalingam, I. Elloumi-Hannachi, S. Ostrovidov and A. Khademhosseini, Acta Biomater., 2011, 7, 1441-1451.

157 J. Park, S. J. Lee, S. Chung, J. H. Lee, W. D. Kim, J. Y. Lee and S. A. Park, Mater. Sci. Eng., C, 2017, 71, 678-684.

158 M. Hassan, K. Dave, R. Chandrawati, F. Dehghani and V. G. Gomes, Eur. Polym. J., 2019, 121, 1-15.

159 J. K. Carrow and A. K. Gaharwar, Macromol. Chem. Phys., 2015, 216, 248-264.

160 A. Bharadwaz and A. C. Jayasuriya, Mater. Sci. Eng., C, 2020, 110, 110698.

161 A. Bibi, S. U. Rehman and A. Yaseen, Mater. Res. Exp., 2019, 6, 092001.

162 J. Venkatesan, I. Bhatnagar, P. Manivasagan, K. H. Kang and S. K. Kim, Int. J. Biol. Macromol., 2015, 72, 269-281.

163 A. Blaeser, N. Million, D. F. D. Campos, L. Gamrad, M. Kopf, C. Rehbock, M. Nachev, B. Sures, S. Barcikowski and H. Fischer, Nano Res., 2016, 9, 3407-3427.

164 J. L. Davila and M. A. d'Avila, Int. J. Adv. Manuf. Technol., 2019, 101, 675-686. 